\title{
Binary Darboux Transformations in Bidifferential Calculus and Integrable Reductions of Vacuum Einstein Equations
}

\author{
Aristophanes DIMAKIS ${ }^{\dagger}$ and Folkert MÜLLER-HOISSEN $\ddagger$ \\ $\dagger$ Department of Financial and Management Engineering, University of the Aegean, \\ 82100 Chios, Greece \\ E-mail:dimakis@aegean.gr \\ ¥Max-Planck-Institute for Dynamics and Self-Organization, 37077 Göttingen, Germany \\ E-mail: folkert.mueller-hoissen@ds.mpg.de
}

Received November 12, 2012, in final form January 29, 2013; Published online February 02, 2013

http://dx.doi.org/10.3842/SIGMA.2013.009

\begin{abstract}
We present a general solution-generating result within the bidifferential calculus approach to integrable partial differential and difference equations, based on a binary Darboux-type transformation. This is then applied to the non-autonomous chiral model, a certain reduction of which is known to appear in the case of the $D$-dimensional vacuum Einstein equations with $D-2$ commuting Killing vector fields. A large class of exact solutions is obtained, and the aforementioned reduction is implemented. This results in an alternative to the well-known Belinski-Zakharov formalism. We recover relevant examples of space-times in dimensions four (Kerr-NUT, Tomimatsu-Sato) and five (single and double Myers-Perry black holes, black saturn, bicycling black rings).
\end{abstract}

Key words: bidifferential calculus; binary Darboux transformation; chiral model; Einstein equations; black ring

2010 Mathematics Subject Classification: 37K10; 16E45

\section{Introduction}

The bidifferential calculus formalism aims to understand integrability features and solutiongenerating methods for (at least a large class of) integrable partial differential or difference equations (PDDEs) resolved from the particularities of examples, i.e., on an as far as possible universal level $[16,17,19]$. The most basic ingredient is a graded associative algebra, supplied with two anti-commuting graded derivations of degree one. It can and should be regarded as a generalization (in the spirit of noncommutative geometry) of the algebra of differential forms on a manifold, but supplied with two analogs of the exterior derivative. Once a PDDE is translated to this framework, it is simple to elaborate its integrability conditions. In fact, it could not be simpler.

In this framework, Darboux transformations (see [35,54,62] and the references therein) have first been addressed in [19]. In the latter work, we had also obtained a very simple solution generating result that evolved into improved versions in recent applications $[15,18,20]$. Its relation with Darboux transformations has been further clarified in [15] (see Appendix A therein), an essential step toward the much more general result that we present in this work. The resulting class of solutions is expressed in a universal way, in the sense that the corresponding formula holds simultaneously for all integrable PDDEs possessing a bidifferential calculus formulation. Choosing a bidifferential calculus associated with a specific PDDE, we can generate infinite families of (soliton-like) solutions. 
In Section 2 we recall some basics of bidifferential calculus and set up the stage in a concise way to formulate our general result about Darboux transformations. This hardly requires previous knowledge and can be taken as an independent and self-contained step into the world of integrable PDDEs. That this is indeed a very powerful tool, is demonstrated in the rest of this work, where we concentrate on one of the more tricky examples of integrable PDEs.

In Section 3 we elaborate in detail the example of the non-autonomous chiral model equation

$$
\left(\rho g_{z} g^{-1}\right)_{z}+\epsilon\left(\rho g_{\rho} g^{-1}\right)_{\rho}=0
$$

for an $m \times m$ matrix $g$, where $\rho>0$ and $z$ are independent real variables and $\epsilon= \pm 1 .^{1}$ With $\epsilon=1$, this governs the case of the stationary, axially symmetric vacuum Einstein $(m=2)$ and Einstein-Maxwell $(m=3)$ equations in four dimensions, where we have two commuting Killing vector fields, one spacelike and the other one asymptotically timelike (see, e.g., $[2,3,48,68]$ ). In an analogous way, (1.1) with $m>3$ appears in the dimensional reduction of (the bosonic part of) higher-dimensional supergravity theories to two dimensions (see, e.g., [5, 25, 30, 36, 45, 73]). With $\epsilon=-1$, the above equation appears in the case of vacuum solutions of Einstein's equations in four dimensions with two commuting spacelike Killing vector fields [2,4,34,77], describing in particular cylindrical gravitational waves.

In Section 3, we also present a reduction condition that, imposed on the obtained family of solutions, achieves that $g$ is symmetric. Any such real and symmetric $g$ determines a solution of the vacuum Einstein equations in $m+2$ dimensions, as recalled in Section 4 . The resulting recipe to construct solutions of the vacuum Einstein equations is close to the familiar BelinskiZakharov method [2-4], which has been applied in numerous publications. In a sense, what we obtained is a kind of matrix version of the latter.

A well-known reformulation of the equations obtained from the integrable reduction of the four-dimensional vacuum Einstein (and also the Einstein-Maxwell) equations by introduction of the so-called twist potential (a crucial step toward the Ernst equation), connects space-time metrics in a different way with the non-autonomous chiral model (see, e.g., [58]). Solutions of the latter are then required to have a constant determinant. This allows a constant seed and thus an application of the restricted solution-generating result in [15] (also see Remark 4.3 below). It does not work in the more direct approach we take in the present work, but here we resolve the restriction in [15].

In Section 4 we elaborate several examples in four and five space-time dimensions, and show that the resulting metrics include important solutions of Einstein's equations, in particular some of the more recently found black objects in five dimensions, which have no counterpart in four dimensions (see, e.g., $[25,42,45,73]$ ). Here we used MathematicA ${ }^{2}$ in a substantial way.

Section 5 contains some concluding remarks.

\section{Binary Darboux transformations in bidifferential calculus}

A graded associative algebra is an associative algebra $\Omega$ over $\mathbb{C}$ with a direct sum decomposition $\Omega=\bigoplus_{r>0} \Omega^{r}$ into a subalgebra $\mathcal{A}:=\Omega^{0}$ and $\mathcal{A}$-bimodules $\Omega^{r}$, such that $\Omega^{r} \Omega^{s} \subseteq \Omega^{r+s}$. A bidifferential calculus (or bidifferential graded algebra) is a unital graded associative algebra $\Omega$, equipped with two (C्C-linear) graded derivations ${ }^{3} \mathrm{~d}, \overline{\mathrm{d}}: \Omega \rightarrow \Omega$ of degree one (hence $\mathrm{d} \Omega^{r} \subseteq \Omega^{r+1}$, $\left.\overline{\mathrm{d}} \Omega^{r} \subseteq \Omega^{r+1}\right)$, with the properties

$$
\mathrm{d}^{2}=\overline{\mathrm{d}}^{2}=\mathrm{d} \overline{\mathrm{d}}+\overline{\mathrm{d}} \mathrm{d}=0 \text {. }
$$

\footnotetext{
${ }^{1}$ A subscript indicates a partial derivative with respect to the corresponding variable. Formally, $\rho \mapsto \mathrm{i} \rho$ relates the two values of $\epsilon$ in (1.1).

${ }^{2}$ Mathematica Edition: Version 8, Wolfram Research, Inc., Champaign, Illinois, 2010.

${ }^{3}$ Hence $\mathrm{d}$ and $\overline{\mathrm{d}}$ both satisfy the graded Leibniz rule $\mathrm{d}\left(\chi \chi^{\prime}\right)=(\mathrm{d} \chi) \chi^{\prime}+(-1)^{r} \chi \mathrm{d} \chi^{\prime}$, for all $\chi \in \Omega^{r}$ and $\chi^{\prime} \in \Omega$.
} 
In the bidifferential calculus approach to integrable PDDEs we are looking for a choice $(\Omega, \mathrm{d}, \overline{\mathrm{d}})$ and some $\phi \in \mathcal{A}$, or some invertible $g \in \mathcal{A}$, such that either

$$
\overline{\mathrm{d}} \mathrm{d} \phi=\mathrm{d} \phi \mathrm{d} \phi
$$

or

$$
\mathrm{d}\left[(\overline{\mathrm{d}} g) g^{-1}\right]=0
$$

is equivalent to a certain PDDE. The two equations are related by the Miura equation

$$
(\overline{\mathrm{d}} g) g^{-1}=\mathrm{d} \phi,
$$

which establishes a kind of Miura transformation ${ }^{4}$ between the two equations (2.2) and (2.3). This equation has both, (2.2) and (2.3), as integrability conditions. As a consequence, if we find a solution pair $(\phi, g)$ of (2.4), then $\phi$ solves (2.2) and $g$ solves (2.3).

In the following theorem, we formulate a solution-generating result which amounts to a transformation that takes a given solution $\left(\phi_{0}, g_{0}\right)$ of the Miura equation (2.4) to a new solution of $(2.4)$,

$$
\left(\phi_{0}, g_{0}\right) \mapsto(\phi, g)
$$

This induces corresponding transformations of solutions of (2.2), respectively (2.3).

Let now $\mathcal{A}$ be the algebra of all finite-dimensional ${ }^{5}$ matrices, with entries in a unital algebra $\mathcal{B}$. The product of two matrices is defined to be zero if the sizes of the two matrices do not match. In the following we assume that there is a graded algebra $\Omega$ with $\Omega^{0}=\mathcal{A}$, and a bidifferential calculus $(\Omega, \mathrm{d}, \overline{\mathrm{d}})$, and such that $\mathrm{d}$ and $\overline{\mathrm{d}}$ preserve the size of matrices. $I=I_{m}$ and $\boldsymbol{I}=\boldsymbol{I}_{n}$ denote the $m \times m$, respectively $n \times n$, identity matrix, and we assume that they are annihilated by $\mathrm{d}$ and $\overline{\mathrm{d}}$. Furthermore, $\operatorname{Mat}(m, n, \mathcal{B})$ denotes the set of $m \times n$ matrices over $\mathcal{B}$.

Theorem 2.1. Let $\phi_{0}, g_{0} \in \operatorname{Mat}(m, m, \mathcal{B})$ solve the Miura equation (2.4). Let $\boldsymbol{P}, \boldsymbol{Q} \in$ $\operatorname{Mat}(n, n, \mathcal{B})$ be invertible solutions of

$$
\overline{\mathrm{d}} \boldsymbol{P}=(\mathrm{d} \boldsymbol{P}) \boldsymbol{P}, \quad \overline{\mathrm{d}} \boldsymbol{Q}=\boldsymbol{Q} \mathrm{d} \boldsymbol{Q},
$$

which are independent in the sense that $\boldsymbol{Q} \boldsymbol{Y}=\boldsymbol{Y} \boldsymbol{P}$ implies $\boldsymbol{Y}=0$. Let $\boldsymbol{U} \in \operatorname{Mat}(m, n, \mathcal{B})$ and $\boldsymbol{V} \in \operatorname{Mat}(n, m, \mathcal{B})$ be solutions of the linear equations ${ }^{6}$

$$
\overline{\mathrm{d}} \boldsymbol{U}=(\mathrm{d} \boldsymbol{U}) \boldsymbol{P}+\left(\mathrm{d} \phi_{0}\right) \boldsymbol{U}, \quad \overline{\mathrm{d}} \boldsymbol{V}=\boldsymbol{Q} \mathrm{d} \boldsymbol{V}-\boldsymbol{V} \mathrm{d} \phi_{0} .
$$

Furthermore, let $\boldsymbol{X} \in \operatorname{Mat}(n, n, \mathcal{B})$ be an invertible solution of the Sylvester-type equation

$$
\boldsymbol{X P}-\boldsymbol{Q X}=\boldsymbol{V U}
$$

Then

$$
\phi=\phi_{0}+\boldsymbol{U} \boldsymbol{X}^{-1} \boldsymbol{V}, \quad g=\left(I+\boldsymbol{U}(\boldsymbol{Q} \boldsymbol{X})^{-1} \boldsymbol{V}\right) g_{0}
$$

solve the Miura equation (2.4), and thus also (2.2), respectively (2.3).

\footnotetext{
${ }^{4}$ The original Miura transformation maps solutions of the modified Korteweg-de Vries equation to solutions of the Korteweg-de Vries equation.

${ }^{5}$ An extension to a suitable class of $\infty$-dimensional matrices, respectively operators, is certainly possible.

${ }^{6}$ If we are primarily interested in solving $(2.3)$, it is more convenient to replace $\mathrm{d} \phi_{0}$ by $\left(\overline{\mathrm{d}} g_{0}\right) g_{0}^{-1}$ in these equations, by use of the Miura equation for $\left(\phi_{0}, g_{0}\right)$.
} 
Proof. Acting on (2.7) with $\overline{\mathrm{d}}$, using the rules of bidifferential calculus, (2.5), (2.6), and (2.7) again, we find

$$
\boldsymbol{Q}[\mathrm{d} \boldsymbol{X}-(\mathrm{d} \boldsymbol{X}) \boldsymbol{P}+(\mathrm{d} \boldsymbol{Q}) \boldsymbol{X}+(\mathrm{d} \boldsymbol{V}) \boldsymbol{U}]=[\overline{\mathrm{d}} \boldsymbol{X}-(\mathrm{d} \boldsymbol{X}) \boldsymbol{P}+(\mathrm{d} \boldsymbol{Q}) \boldsymbol{X}+(\mathrm{d} \boldsymbol{V}) \boldsymbol{U}] \boldsymbol{P} .
$$

Since $\boldsymbol{P}$ and $\boldsymbol{Q}$ are assumed to be independent, this implies ${ }^{7}$

$$
\overline{\mathrm{d}} \boldsymbol{X}-(\mathrm{d} \boldsymbol{X}) \boldsymbol{P}+(\mathrm{d} \boldsymbol{Q}) \boldsymbol{X}+(\mathrm{d} \boldsymbol{V}) \boldsymbol{U}=0 .
$$

Using also (2.5) and (2.7), we easily deduce that

$$
\begin{aligned}
\overline{\mathrm{d}}(\boldsymbol{Q} \boldsymbol{X})^{-1} & =-\boldsymbol{X}^{-1}\left[(\mathrm{~d} \boldsymbol{X}) \boldsymbol{X}^{-1}(\boldsymbol{X} \boldsymbol{P})-(\mathrm{d} \boldsymbol{V}) \boldsymbol{U}\right](\boldsymbol{Q} \boldsymbol{X})^{-1} \\
& =\left(\mathrm{d} \boldsymbol{X}^{-1}\right)\left[\boldsymbol{I}+\boldsymbol{V} \boldsymbol{U}(\boldsymbol{Q} \boldsymbol{X})^{-1}\right]+\boldsymbol{X}^{-1}(\mathrm{~d} \boldsymbol{V}) \boldsymbol{U}(\boldsymbol{Q} \boldsymbol{X})^{-1}
\end{aligned}
$$

This is then used in the elaboration of

$$
\overline{\mathrm{d}} g=\overline{\mathrm{d}}\left[\left(I+\boldsymbol{U}(\boldsymbol{Q} \boldsymbol{X})^{-1} \boldsymbol{V}\right) g_{0}\right]
$$

via the graded derivation (Leibniz) rule for $\overline{\mathrm{d}}$. We eliminate all further terms involving a $\overline{\mathrm{d}}$ with the help of (2.6), and apply (2.7) to eliminate a $\boldsymbol{P}$ in favor of a $\boldsymbol{Q}$. Finally we obtain $\overline{\mathrm{d}} g=(\mathrm{d} \phi) g$ with $\phi$ given by (2.8). Our assumptions ensure that the inverse of $g$ exists. It is given by

$$
g^{-1}=g_{0}^{-1}\left(I-\boldsymbol{U}(\boldsymbol{X} \boldsymbol{P})^{-1} \boldsymbol{V}\right) .
$$

The integrability conditions of (2.6) and (2.9) are satisfied as a consequence of (2.5) and $\overline{\mathrm{d}} \mathrm{d} \phi_{0}=$ $\mathrm{d} \phi_{0} \mathrm{~d} \phi_{0}$ (which follows from $\left.\left(\overline{\mathrm{d}} g_{0}\right) g_{0}^{-1}=\mathrm{d} \phi_{0}\right)$.

For a reader acquainted with corresponding results about binary Darboux transformations in the literature on integrable systems, the similarity will be evident (see, e.g., $[12,35,54,59,62]$ ). It is close to results in $[63-65]^{8}$. A new feature, however, is the dependence of the linear equations (2.6) on solutions $\boldsymbol{P}$ and $\boldsymbol{Q}$ of the nonlinear equations (2.5). This generalization is crucial for our application to the Einstein equations. As already mentioned in the introduction, Theorem 2.1 considerably generalizes previous, more restricted results, see in particular [15].

Remark 2.1. As a consequence of (2.5), $\boldsymbol{P}$ and $-\boldsymbol{Q}$ solve the $n \times n$ version of (2.2). Since we assume that they are invertible, $\boldsymbol{P}$ and $\boldsymbol{Q}^{-1}$ solve the $n \times n$ version of (2.3). If $\boldsymbol{U}$ is a solution of the first of (2.6), then also $\boldsymbol{U} \boldsymbol{P}$, by use of the first of (2.5). If $\boldsymbol{V}$ is a solution of the second of (2.6), then also $\boldsymbol{Q} \boldsymbol{V}$, by use of the second of (2.5).

Remark 2.2. By direct computations one verifies the following "scaling property". Let $\boldsymbol{U}$ and $\boldsymbol{V}$ solve (2.6) and let

$$
\hat{g}_{0}=w g_{0}, \quad \hat{\boldsymbol{U}}=\boldsymbol{U} \boldsymbol{W}_{1}, \quad \hat{\boldsymbol{V}}=\boldsymbol{W}_{2} \boldsymbol{V},
$$

with a solution $(\varphi, w)$ of the $m=1$ version of $(2.4)$, and invertible $\boldsymbol{W}_{i} \in \operatorname{Mat}(n, n, \mathcal{B})$, subject to

$$
\left[\boldsymbol{W}_{1}, \boldsymbol{P}\right]=0, \quad\left[\boldsymbol{W}_{2}, \boldsymbol{Q}\right]=0 .
$$

We further assume that $\varphi$ and $w$ are in the center of $\Omega .{ }^{9}$ If $\boldsymbol{W}_{1}$ and $\boldsymbol{W}_{2}$ satisfy

$$
\overline{\mathrm{d}} \boldsymbol{W}_{1}=\left(\mathrm{d} \boldsymbol{W}_{1}\right) \boldsymbol{P}+(\overline{\mathrm{d}} w) w^{-1} \boldsymbol{W}_{1}, \quad \overline{\mathrm{d}} \boldsymbol{W}_{2}=\boldsymbol{Q} \mathrm{d} \boldsymbol{W}_{2}-\boldsymbol{W}_{2}(\overline{\mathrm{d}} w) w^{-1},
$$

\footnotetext{
${ }^{7}$ It is a key feature of Theorem 2.1 that this equation is automatically solved if $\boldsymbol{P}$ and $\boldsymbol{Q}$ are independent. Relaxing the latter assumption, the theorem remains valid if this equation is added to the assumptions.

${ }^{8}$ The precise relation has still to be clarified.

${ }^{9}$ This holds, e.g., in the case of the non-autonomous chiral model treated in Section 3. But this assumption will be a (perhaps too) severe restriction if $\mathcal{B}$ involves differential or difference operators.
} 
then $\hat{\boldsymbol{U}}$ and $\hat{\boldsymbol{V}}$ solve (2.6), with $\phi_{0}$ replaced by $\hat{\phi}_{0}=\phi_{0}+\varphi I$. Furthermore, $\hat{\boldsymbol{X}}=\boldsymbol{W}_{2} \boldsymbol{X} \boldsymbol{W}_{1}$ solves (2.7) with $\boldsymbol{U}, \boldsymbol{V}$ replaced by $\hat{\boldsymbol{U}}, \hat{\boldsymbol{V}}$. For the new solutions of (2.4) (and thus (2.2), respectively (2.3)), determined by the theorem, we find

$$
\hat{\phi}=\phi+\varphi I, \quad \hat{g}=w g,
$$

where $\phi$ and $g$ are given by (2.8). Hence, under the stated conditions, multiplication of $g_{0}$ by a "scalar" solution $w$ of (2.3) simplify amounts to multiplication of $g$ by $w$. In the special case where $w$ is the identity element and $g$ thus remains unchanged, the above equations for $\boldsymbol{W}_{1}$ and $\boldsymbol{W}_{2}$ still allow non-trivial transformations of $\boldsymbol{U}$ and $\boldsymbol{V}$. These results will be used in Section 4 .

Remark 2.3. Let $\left(\phi_{i}, g_{i}\right), i=1,2$, be two solutions obtained via Theorem 2.1 from the same seed solution $\left(\phi_{0}, g_{0}\right)$. Let $\left(\boldsymbol{P}_{i}, \boldsymbol{Q}_{i}, \boldsymbol{U}_{i}, \boldsymbol{V}_{i}\right)$ be corresponding solutions of (2.5) and (2.6). Then

$$
\boldsymbol{P}=\left(\begin{array}{cc}
\boldsymbol{P}_{1} & 0 \\
0 & \boldsymbol{P}_{2}
\end{array}\right), \quad \boldsymbol{Q}=\left(\begin{array}{cc}
\boldsymbol{Q}_{1} & 0 \\
0 & \boldsymbol{Q}_{2}
\end{array}\right), \quad \boldsymbol{U}=\left(\boldsymbol{U}_{1}, \boldsymbol{U}_{2}\right), \quad \boldsymbol{V}=\left(\begin{array}{l}
\boldsymbol{V}_{1} \\
\boldsymbol{V}_{2}
\end{array}\right)
$$

also solve (2.5) and (2.6). If $\boldsymbol{P}$ and $\boldsymbol{Q}$ are independent and if (2.7) has an invertible solution $\boldsymbol{X}$, then (2.8) determines a new solution of (2.4), and thus new solutions of (2.2) and (2.3). This expresses a nonlinear superposition principle. By iteration, one can build superpositions of an arbitrary number of "elementary" solutions, hence (analogs of) "multi-solitons".

Remark 2.4. We note that the first of equations (2.6) is a special case (and $n \times n$ matrix version) of the general linear equation

$$
\overline{\mathrm{d}} \psi-\mathbb{A} \psi=\mathrm{d} \psi P, \quad \text { where } \quad \mathbb{A}=\mathrm{d} \phi .
$$

Here $P$ is a solution of the nonlinear equation

$$
\overline{\mathrm{d}} P=(\mathrm{d} P) P .
$$

An $n \times n$ matrix version of it appears in (2.5). The integrability condition of (2.10) is (2.2). If we set $\mathbb{A}=(\overline{\mathrm{d}} g) g^{-1}$, the integrability condition is (2.3) instead. A similar statement holds for the second of equations (2.6), the "adjoint linear system". The Darboux transformation (2.8) is "binary" since it involves solutions of the linear system as well as of the adjoint.

Remark 2.5. In the special case where $\Omega$ is the algebra of matrix-valued differential forms on a manifold, and d the exterior derivative, any tensor field $\mathcal{N}$ of type $(1,1)$ with vanishing Nijenhuis torsion determines a map $\overline{\mathrm{d}}_{\mathcal{N}}$ satisfying the above conditions [7,31]. Moreover, according to Frölicher-Nijenhuis theory [31], any $\overline{\mathrm{d}}$ such that (2.1) holds has to be of this form. Finite-dimensional integrable systems have been considered in this framework in [13]. The generalization to Lie algebroid structures is nicely described in [6]. See also [1,51,52] for related aspects. We should stress, however, that in our central examples we depart from differential geometry and consider a differential calculus in a weaker sense (e.g., of noncommutative geometry).

The setting of the above theorem allows in principle structures far away from classical calculus and in particular differential geometry, then dealing with equations beyond differential and difference equations. A somewhat more restricted framework is given by setting

$$
\Omega=\mathcal{A} \otimes \bigwedge\left(\mathbb{C}^{N}\right)
$$

where $\bigwedge\left(\mathbb{C}^{N}\right)$ denotes the exterior (Grassmann) algebra of the vector space $\mathbb{C}^{N}$. In this case it is sufficient to define suitable operators $\mathrm{d}$ and $\overline{\mathrm{d}}$ on $\mathcal{A}$, since they extend to $\Omega$ in an evident way. Many integrable PDDEs have been treated in this framework and in the next section we turn to an important example. 


\section{Solutions of the non-autonomous chiral model}

The non-autonomous chiral model is well-known to be a reduction of the (anti-) self-dual YangMills equations, for which a very simple bidifferential calculus exists [19] (that may actually be considered as a prototype). From the latter one can then derive a bidifferential calculus for (1.1). It is determined by

$$
\mathrm{d} f=-f_{z} \xi_{1}+e^{\theta}\left(f_{\rho}-\rho^{-1} f_{\theta}\right) \xi_{2}, \quad \overline{\mathrm{d}} f=e^{-\theta}\left(f_{\rho}+\rho^{-1} f_{\theta}\right) \xi_{1}+\epsilon f_{z} \xi_{2}
$$

for $f \in C^{\infty}\left(\mathbb{R}^{3}\right)$ (cf. [15]). $\xi_{1}, \xi_{2}$ is a basis of $\bigwedge^{1}\left(\mathbb{C}^{2}\right)$. Choosing $\mathcal{B}=C^{\infty}\left(\mathbb{R}^{3}\right)$, d and $\overline{\mathrm{d}}$ extend to $\mathcal{A} \otimes \wedge\left(\mathbb{C}^{2}\right)$ via $\mathrm{d}\left(f_{1} \xi_{1}+f_{2} \xi_{2}\right)=\left(\mathrm{d} f_{1}\right) \wedge \xi_{1}+\left(\mathrm{d} f_{2}\right) \wedge \xi_{2}$ and $\mathrm{d}\left(f \xi_{1} \wedge \xi_{2}\right)=(\mathrm{d} f) \wedge \xi_{1} \wedge \xi_{2}=0$, and correspondingly for $\overline{\mathrm{d}}$. For an $m \times m$ matrix-valued function $g,(2.3)$ now takes the form

$$
\left(\rho g_{z} g^{-1}\right)_{z}+\epsilon\left(\rho g_{\rho} g^{-1}\right)_{\rho}-\left[\left(g_{\rho}+\rho^{-1} g_{\theta}\right) g^{-1}\right]_{\theta}+\left(g_{\theta} g^{-1}\right)_{\rho}=0
$$

which reduces to (1.1) if $g$ does not depend on $\theta$. The coordinate $\theta$ is needed to have the properties of a bidifferential calculus, but addressing (1.1) we are primarily interested in equations for objects that do not depend on it.

Using this bidifferential calculus, the Miura equation (2.4) decomposes into

$$
\left(g_{\rho}+\rho^{-1} g_{\theta}\right) g^{-1}=-e^{\theta} \phi_{z}, \quad g_{z} g^{-1}=\epsilon e^{\theta}\left(\phi_{\rho}-\rho^{-1} \phi_{\theta}\right) .
$$

If $g$ is $\theta$-independent, this requires

$$
\phi=e^{-\theta} \tilde{\phi}
$$

with $\theta$-independent $\tilde{\phi}$, and then reduces to

$$
g_{\rho} g^{-1}=-\tilde{\phi}_{z}, \quad \epsilon g_{z} g^{-1}=\tilde{\phi}_{\rho}+\rho^{-1} \tilde{\phi} .
$$

In the following, we elaborate Theorem 2.1 using the above bidifferential calculus. Section 3.1 provides the complete solution of the two nonlinear equations (2.5) under the condition that $\boldsymbol{P}$ (respectively $\boldsymbol{Q}$ ) has geometrically simple spectrum (i.e., for each eigenvalue there is a unique corresponding Jordan block in the Jordan normal form), also see [15]. In this case the two equations actually coincide. Then it only remains to solve linear equations, see Section 3.2 and Section 3.3 below. In Section 3.4 we present a condition to be imposed on the data in order to achieve that the solution $g$ is symmetric (or Hermitian). This reduction is crucial in the context of Einstein's equations, see Section 4. Most of the following is, however, independent of additional assumptions and provides a general procedure to construct solutions of the nonautonomous chiral model. The corresponding equations cannot be solved explicitly without some restrictions, in particular on the form of the seed solution. We content ourselves with providing illustrative and important examples relevant in the context of gravity in Section 4.

\subsection{The equations for $P$ and $Q$}

In terms of

$$
\tilde{\boldsymbol{P}}:=e^{\theta} \boldsymbol{P},
$$

the first of the two equations (2.5) decomposes into the following pair of equations,

$$
\tilde{\boldsymbol{P}}_{\rho}+\rho^{-1}\left(\tilde{\boldsymbol{P}}_{\theta}-\tilde{\boldsymbol{P}}\right)=-\tilde{\boldsymbol{P}}_{z} \tilde{\boldsymbol{P}}, \quad \epsilon \tilde{\boldsymbol{P}}_{z}=\left[\tilde{\boldsymbol{P}}_{\rho}-\rho^{-1}\left(\tilde{\boldsymbol{P}}_{\theta}-\tilde{\boldsymbol{P}}\right)\right] \tilde{\boldsymbol{P}},
$$


which are autonomous in the variable $\theta$. Assuming that $\tilde{\boldsymbol{P}}$ and $\boldsymbol{I}+\epsilon \tilde{\boldsymbol{P}}^{2}$ are invertible, and that $\tilde{\boldsymbol{P}}$ is $\theta$-independent, (3.3) implies

$$
\tilde{\boldsymbol{P}}^{2}-2 \rho^{-1}(z \boldsymbol{I}-\boldsymbol{A}) \tilde{\boldsymbol{P}}-\epsilon \boldsymbol{I}=0,
$$

with an arbitrary constant $n \times n$ matrix $\boldsymbol{A}$ (also see [15]). This is a matrix version of the pole trajectories in the Belinski-Zakharov approach $[2,4]$. A well-known symmetry of the latter extends to the matrix case: (3.4) is invariant under $\tilde{\boldsymbol{P}} \mapsto-\epsilon \tilde{\boldsymbol{P}}^{-1}$. For the following result, see Lemma 4.1 in [15].

Lemma 3.1. Any $\theta$-independent, invertible solution of (3.4), which commutes with its derivatives with respect to $\rho$ and $z$, solves (3.3).

$\boldsymbol{A}$ can be taken in Jordan normal form $\boldsymbol{A}=\operatorname{block}-\operatorname{diag}\left(\boldsymbol{A}_{n_{1}}, \ldots, \boldsymbol{A}_{n_{s}}\right)$, without restriction of generality, and a solution of (3.4) is then given by

$$
\tilde{\boldsymbol{P}}=\operatorname{block}-\operatorname{diag}\left(\tilde{\boldsymbol{P}}_{n_{1}}, \ldots, \tilde{\boldsymbol{P}}_{n_{s}}\right),
$$

where the block $\tilde{\boldsymbol{P}}_{n_{i}}$ is a solution of (3.4) with $\boldsymbol{A}$ replaced by the Jordan block $\boldsymbol{A}_{n_{i}}$, see the next examples. Under the assumption that $\tilde{\boldsymbol{P}}$ has geometrically simple spectrum, this is the most general solution of $\overline{\mathrm{d}} \boldsymbol{P}=(\mathrm{d} \boldsymbol{P}) \boldsymbol{P}$ with $\theta$-independent $\tilde{\boldsymbol{P}}=e^{\theta} \boldsymbol{P}[15]$.

Example 3.1. If $\boldsymbol{A}$ is diagonal, i.e., $\boldsymbol{A}=\operatorname{diag}\left(a_{1}, \ldots, a_{n}\right)$ with constants $a_{i}$, corresponding solutions of $(3.4)$ are given by $\tilde{\boldsymbol{P}}=\operatorname{diag}\left(\tilde{p}_{1}, \ldots, \tilde{p}_{n}\right)$, where $\tilde{p}_{i}$ is any of

$$
p_{i}=\rho^{-1}\left(z-a_{i}+\mathfrak{R}_{i}\right), \quad \bar{p}_{i}=\rho^{-1}\left(z-a_{i}-\mathfrak{R}_{i}\right),
$$

with $\mathfrak{R}_{i}=\sqrt{\left(z-a_{i}\right)^{2}+\epsilon \rho^{2}}$. Note that $\bar{p}_{i}=-\epsilon / p_{i}$.

Remark 3.1. For better comparison with the relevant literature, we will sometimes write

$$
p_{i}=\frac{\rho}{\mu_{i}}, \quad \bar{p}_{i}=\frac{\rho}{\bar{\mu}_{i}},
$$

where

$$
\mu_{i}=\sqrt{\left(z-a_{i}\right)^{2}+\epsilon \rho^{2}}-\left(z-a_{i}\right), \quad \bar{\mu}_{i}=-\sqrt{\left(z-a_{i}\right)^{2}+\epsilon \rho^{2}}-\left(z-a_{i}\right) .
$$

$\mu_{i}$ and $\bar{\mu}_{i}$ are often referred to as (indicating the presence of) a soliton and an anti-soliton, respectively. Note that $\bar{\mu}_{i}=-\epsilon \rho^{2} / \mu_{i}$. If $\epsilon=1$, then $\mu_{i}$ is non-negative and only vanishes on a subset of $\{\rho=0\}$.

Example 3.2. For an $r \times r$ Jordan block

$$
\boldsymbol{A}_{r}=a \boldsymbol{I}_{r}+\boldsymbol{N}_{r}, \quad \boldsymbol{N}_{r}=\left(\begin{array}{ccccc}
0 & 1 & 0 & \cdots & 0 \\
0 & 0 & 1 & \ddots & \vdots \\
\vdots & & \ddots & \ddots & \vdots \\
\vdots & & & \ddots & 1 \\
0 & \cdots & \cdots & \cdots & 0
\end{array}\right)
$$

(3.4) has the solutions (see [15])

$$
\tilde{\boldsymbol{P}}_{r}=\rho^{-1}\left(z \boldsymbol{I}_{r}-\boldsymbol{A}_{r}+\sum_{k=0}^{r-1}\left(\begin{array}{c}
1 / 2 \\
k
\end{array}\right)( \pm \mathfrak{R})^{1-2 k}\left[2(a-z) \boldsymbol{N}_{r}+\boldsymbol{N}_{r}^{2}\right]^{k}\right)
$$


where $\mathfrak{R}=\sqrt{(z-a)^{2}+\epsilon \rho^{2}}$. This is an upper-triangular Toeplitz matrix and thus commutes with its derivatives. In particular, we have $\tilde{\boldsymbol{P}}_{1}=\tilde{p}=\rho^{-1}[z-a \pm \mathfrak{R}]$ and

$$
\tilde{\boldsymbol{P}}_{2}=\tilde{p}\left(\begin{array}{cc}
1 & \mp \mathfrak{R}^{-1} \\
0 & 1
\end{array}\right), \quad \tilde{\boldsymbol{P}}_{3}=\tilde{p}\left(\begin{array}{ccc}
1 & \mp \mathfrak{R}^{-1} & \pm \frac{\epsilon}{2} \rho \mathfrak{R}^{-3} \tilde{p}^{-1} \\
0 & 1 & \mp \mathfrak{R}^{-1} \\
0 & 0 & 1
\end{array}\right)
$$

For the solutions $\tilde{\boldsymbol{P}}$ obtained in this way, and thus $\boldsymbol{P}$, we have

$$
\overline{\mathrm{d}} \boldsymbol{P}=(\mathrm{d} \boldsymbol{P}) \boldsymbol{P}=\boldsymbol{P}(\mathrm{d} \boldsymbol{P}),
$$

so that they also provide us with solutions $\boldsymbol{Q}=e^{-\theta} \tilde{\boldsymbol{Q}}$, with $\theta$-independent $\tilde{\boldsymbol{Q}}$, of the second of $(2.5)$, also see [15].

Recall that on the way to the above results we assumed that $\tilde{\boldsymbol{P}}$ and $\boldsymbol{I}+\epsilon \tilde{\boldsymbol{P}}^{2}$ are invertible, and then also $\tilde{\boldsymbol{Q}}$ and $\boldsymbol{I}+\epsilon \tilde{\boldsymbol{Q}}^{2}$. These assumption will also be made throughout in the following.

Remark 3.2. The source matrix $\boldsymbol{A}$ for $\tilde{\boldsymbol{P}}$ and the corresponding source matrix $\boldsymbol{A}^{\prime}$ for $\tilde{\boldsymbol{Q}}$ can be assumed to be simultaneously in Jordan normal form, without restriction of generality. Since this is achieved by similarity transformations with constant transformation matrices, the latter can be absorbed by redefinitions that restore all the equations obtained from Theorem 2.1. But in general this will no longer be so if we impose a reduction condition that relates $\tilde{\boldsymbol{P}}$ and $\tilde{\boldsymbol{Q}}$, as in Section 3.4 below.

\subsection{The equations for $U$ and $V$}

Setting $\phi_{0}=e^{-\theta} \tilde{\phi}_{0}$ with a $\theta$-independent $\tilde{\phi}_{0}$ (cf. (3.1)), (2.6) is autonomous in the variable $\theta$. Assuming that $\boldsymbol{U}, \boldsymbol{V}$ are $\theta$-independent, and using the fact that $\tilde{\phi}_{0}$ has to solve the Miura equations (3.2) together with some $g_{0}$, we obtain the following systems of linear differential equations for $\boldsymbol{U}$ and $\boldsymbol{V}$,

$$
\begin{aligned}
& \boldsymbol{U}_{\rho}=\left(g_{0, \rho} g_{0}^{-1} \boldsymbol{U}-g_{0, z} g_{0}^{-1} \boldsymbol{U} \tilde{\boldsymbol{P}}\right)\left(\boldsymbol{I}+\epsilon \tilde{\boldsymbol{P}}^{2}\right)^{-1}, \\
& \boldsymbol{U}_{z}=\left(g_{0, z} g_{0}^{-1} \boldsymbol{U}+\epsilon g_{0, \rho} g_{0}^{-1} \boldsymbol{U} \tilde{\boldsymbol{P}}\right)\left(\boldsymbol{I}+\epsilon \tilde{\boldsymbol{P}}^{2}\right)^{-1},
\end{aligned}
$$

and

$$
\begin{aligned}
& \boldsymbol{V}_{\rho}=\left(\boldsymbol{I}+\epsilon \tilde{\boldsymbol{Q}}^{2}\right)^{-1}\left(\tilde{\boldsymbol{Q}} \boldsymbol{V} g_{0, z} g_{0}^{-1}-\boldsymbol{V} g_{0, \rho} g_{0}^{-1}\right) \\
& \boldsymbol{V}_{z}=-\left(\boldsymbol{I}+\epsilon \tilde{\boldsymbol{Q}}^{2}\right)^{-1}\left(\boldsymbol{V} g_{0, z} g_{0}^{-1}+\epsilon \tilde{\boldsymbol{Q}} \boldsymbol{V} g_{0, \rho} g_{0}^{-1}\right)
\end{aligned}
$$

These equations have to be solved for the given seed solution $g_{0}$ of (2.3). For diagonal $g_{0}$, this is done in the next example for the $\boldsymbol{V}$-equations. Similar results are easily obtained for the $\boldsymbol{U}$-equations.

Example 3.3. Let

$$
\tilde{\boldsymbol{Q}}=\operatorname{diag}\left(\tilde{p}_{1}, \ldots, \tilde{p}_{n}\right), \quad g_{0}=\operatorname{diag}\left(w_{1}, \ldots, w_{m}\right),
$$

where $\tilde{p}_{i}$ is either $p_{i}$ or $\bar{p}_{i}$ in (3.5), and $w_{\alpha}$ is a non-vanishing solution of the scalar (i.e., $m=1$ ) version

$$
\left[\rho(\ln w)_{\rho}\right]_{\rho}=-\epsilon\left[\rho(\ln w)_{z}\right]_{z}
$$


of the non-autonomous chiral model (1.1). Writing $\boldsymbol{V}=\left(V_{i \alpha}\right)$, where $i=1, \ldots, n$ and $\alpha=$ $1, \ldots, m,(3.8)$ reads

$$
\begin{aligned}
& \left(\ln V_{i \alpha}\right)_{\rho}=\frac{1}{1+\epsilon \tilde{p}_{i}^{2}}\left(\tilde{p}_{i}\left(\ln w_{\alpha}\right)_{z}-\left(\ln w_{\alpha}\right)_{\rho}\right), \\
& \left(\ln V_{i \alpha}\right)_{z}=-\frac{1}{1+\epsilon \tilde{p}_{i}^{2}}\left(\left(\ln w_{\alpha}\right)_{z}+\epsilon \tilde{p}_{i}\left(\ln w_{\alpha}\right)_{\rho}\right) .
\end{aligned}
$$

Let us list some simple solutions. If $w_{\alpha}$ is constant, then also $V_{i \alpha}$. If $\tilde{p}_{i}=p_{i}$, then

$$
V_{i \alpha}=k_{i \alpha} \begin{cases}\left(\rho p_{i}\right)^{-1 / 2} & \text { if } w_{\alpha}=\rho \\ \left(\rho p_{i}\right)^{1 / 2}\left(1+\epsilon p_{i}^{-1} p_{\alpha}^{-1}\right) & \text { if } w_{\alpha}=p_{\alpha}\end{cases}
$$

where $p_{\alpha}$ shall be given by the same expression as some $p_{i}$, but with in general different constant, say $a_{\alpha}^{\prime} \cdot k_{i \alpha}$ is an arbitrary constant. If $\tilde{p}_{i}=\bar{p}_{i}$, then

$$
V_{i \alpha}=k_{i \alpha} \begin{cases}\left(p_{i} / \rho\right)^{1 / 2} & \text { if } w_{\alpha}=\rho, \\ \left(p_{i} / \rho\right)^{1 / 2}\left(1+\epsilon p_{i} p_{\alpha}\right)^{-1} & \text { if } w_{\alpha}=p_{\alpha} .\end{cases}
$$

More complicated solutions are now obtained by noting the following.

- If $V_{i \alpha}$ is a solution for $w_{\alpha}$, then $V_{i \alpha}^{-1}$ is a solution for $w_{\alpha}^{-1}$.

- If $w_{\alpha}$ is the product of two solutions of (3.9), then $V_{i \alpha}$ is the product of the respective solutions for the factors.

\subsection{The Sylvester equation and the solution formula}

Recalling that $\boldsymbol{U}, \boldsymbol{V}$ and $\tilde{\boldsymbol{Q}}$ are $\theta$-independent, the formula for ( $\theta$-independent) $g$ in (2.8) requires $\boldsymbol{X}=e^{\theta} \tilde{\boldsymbol{X}}$ with $\theta$-independent $\tilde{\boldsymbol{X}}$. (2.7) becomes the $\theta$-independent Sylvester equation

$$
\tilde{\boldsymbol{X}} \tilde{\boldsymbol{P}}-\tilde{\boldsymbol{Q}} \tilde{\boldsymbol{X}}=\boldsymbol{V U}
$$

If $\operatorname{spec}(\tilde{\boldsymbol{P}}) \cap \operatorname{spec}(\tilde{\boldsymbol{Q}})=\varnothing$, then (3.10) has a unique solution, for any choice of the matrices on the right hand side. The two matrices $\boldsymbol{P}$ and $\boldsymbol{Q}$ are then independent, hence Theorem 2.1 implies that ${ }^{10}$

$$
g=\left(I+\boldsymbol{U}(\tilde{\boldsymbol{Q}} \tilde{\boldsymbol{X}})^{-1} \boldsymbol{V}\right) g_{0}
$$

solves the non-autonomous chiral model equation (1.1). Obviously, scaling $\boldsymbol{U}$ or $\boldsymbol{V}$ with an arbitrary non-zero constant leaves $g$ invariant. We recall from [15] (see Remark 4.4 therein) that

$$
\operatorname{det} g=\frac{\operatorname{det} \tilde{\boldsymbol{P}}}{\operatorname{det} \tilde{\boldsymbol{Q}}} \operatorname{det} g_{0} .
$$

Example 3.4. Let $\tilde{\boldsymbol{P}}$ be diagonal, as in Example 3.1, and also $\tilde{\boldsymbol{Q}}$, with eigenvalues $\tilde{q}_{i}$, given by an expression of the same form as $\tilde{p}_{i}$. If they have no eigenvalue in common, i.e., $\left\{\tilde{p}_{i}\right\} \cap\left\{\tilde{q}_{i}\right\}=\varnothing$, then the unique solution of (3.10) is given by the Cauchy-like matrix

$$
\tilde{\boldsymbol{X}}_{i j}=\frac{(\boldsymbol{V} \boldsymbol{U})_{i j}}{\tilde{p}_{j}-\tilde{q}_{i}}
$$

\footnotetext{
${ }^{10}$ We have to choose $\tilde{\boldsymbol{Q}}$ invertible and make sure that the solution $\tilde{\boldsymbol{X}}$ of (3.10) is invertible. See [14,38] for conditions that guarantee the latter.
} 
A vast literature exists on solutions of the Sylvester equation (3.10), more generally with non-diagonal matrices $\tilde{\boldsymbol{P}}$ and $\tilde{\boldsymbol{Q}}$ (and not necessarily satisfying the spectrum condition that guarantees a unique solution).

Proposition 3.1 ( $[14,37,44])$. Let $\operatorname{spec}(\tilde{\boldsymbol{P}}) \cap \operatorname{spec}(\tilde{\boldsymbol{Q}})=\varnothing$ and

$$
\mathfrak{P}(\lambda)=\sum_{k=0}^{n} \mathfrak{P}_{k} \lambda^{k}
$$

be the characteristic polynomial of $\tilde{\boldsymbol{P}}$. Then the unique solution of the Sylvester equation (3.10) is given by

$$
\tilde{\boldsymbol{X}}=-\mathfrak{P}(\tilde{\boldsymbol{Q}})^{-1} \sum_{k=1}^{n} \mathfrak{P}_{k} \sum_{i=0}^{k-1} \tilde{\boldsymbol{Q}}^{k-1-i} \boldsymbol{V} \boldsymbol{U} \tilde{\boldsymbol{P}}^{i}
$$

Remark 3.3. (2.9) takes the form

$$
\begin{aligned}
& \tilde{\boldsymbol{X}}_{\rho}+\rho^{-1} \tilde{\boldsymbol{X}}+\tilde{\boldsymbol{X}}_{z} \tilde{\boldsymbol{P}}-\tilde{\boldsymbol{Q}}_{z} \tilde{\boldsymbol{X}}-\boldsymbol{V}_{z} \boldsymbol{U}=0, \\
& \epsilon \tilde{\boldsymbol{X}}_{z}-\left(\tilde{\boldsymbol{X}}_{\rho}-\rho^{-1} \tilde{\boldsymbol{X}}\right) \tilde{\boldsymbol{P}}+\left(\tilde{\boldsymbol{Q}}_{\rho}+\rho^{-1} \tilde{\boldsymbol{Q}}\right) \tilde{\boldsymbol{X}}+\boldsymbol{V}_{\rho} \boldsymbol{U}=0 .
\end{aligned}
$$

If we drop the spectrum condition for $\tilde{\boldsymbol{P}}$ and $\tilde{\boldsymbol{Q}}$, these equations also have to be solved. Otherwise they are a consequence of our assumptions (see the proof of Theorem 2.1). (3.14) will only be used in the proof of Proposition 4.1 in Appendix B.

Remark 3.4. Using the above results, we also obtain solutions $\phi$ of (2.2), given by the expression in (2.8), which in the case under consideration and via (3.1) takes the form

$$
\epsilon \tilde{\phi}_{z z}+\left(\tilde{\phi}_{\rho}+\rho^{-1} \tilde{\phi}\right)_{\rho}=\left[\tilde{\phi}_{\rho}+\rho^{-1} \tilde{\phi}, \tilde{\phi}_{z}\right]
$$

(which corrects a typo in (4.3) of [15]).

\subsection{A reduction condition}

It is of particular interest (see Section 4) to find a convenient condition which guarantees that the solution matrix $g$ given by (3.11) is symmetric, i.e., $g^{\top}=g$, where ${ }^{\top}$ means matrix transpose. The following result is easily verified by a direct computation.

Lemma 3.2. Let $\tilde{\boldsymbol{P}}^{\top}=-\epsilon \tilde{\boldsymbol{Q}}^{-1}$ and $g_{0}^{\top}=g_{0}$. If $\boldsymbol{V}$ solves $(3.8)$, then $\boldsymbol{U}=\left(\boldsymbol{V} g_{0}\right)^{\top}$ solves (3.7).

Proposition 3.2. Let $\tilde{\boldsymbol{P}}^{\top}=-\epsilon \tilde{\boldsymbol{Q}}^{-1}, \operatorname{spec}(\tilde{\boldsymbol{P}}) \cap \operatorname{spec}(\tilde{\boldsymbol{Q}})=\varnothing, g_{0}^{\top}=g_{0}$, and $\boldsymbol{U}=\left(\boldsymbol{V} g_{0}\right)^{\top}$. Then $g$ given by (3.11) is symmetric.

Proof. Using $g_{0}^{\top}=g_{0}$ and $\boldsymbol{U}=\left(\boldsymbol{V} g_{0}\right)^{\top}$, the Sylvester equation (3.10) and its transpose lead to

$$
\tilde{\boldsymbol{Q}}\left[\tilde{\boldsymbol{Q}} \tilde{\boldsymbol{X}}-(\tilde{\boldsymbol{Q}} \tilde{\boldsymbol{X}})^{\top}\right]=\tilde{\boldsymbol{Q}}\left[\tilde{\boldsymbol{X}} \tilde{\boldsymbol{P}}-\tilde{\boldsymbol{P}}^{\top} \tilde{\boldsymbol{X}}^{\top}\right]=\left[\tilde{\boldsymbol{Q}} \tilde{\boldsymbol{X}}+\epsilon \tilde{\boldsymbol{X}}^{\top} \tilde{\boldsymbol{P}}^{-1}\right] \tilde{\boldsymbol{P}}=\left[\tilde{\boldsymbol{Q}} \tilde{\boldsymbol{X}}-(\tilde{\boldsymbol{Q}} \tilde{\boldsymbol{X}})^{\top}\right] \tilde{\boldsymbol{P}}
$$

In the last two steps we used $\tilde{\boldsymbol{P}}^{\top}=-\epsilon \tilde{\boldsymbol{Q}}^{-1}$. Now the spectrum condition implies $\tilde{\boldsymbol{Q}} \tilde{\boldsymbol{X}}=(\tilde{\boldsymbol{Q}} \tilde{\boldsymbol{X}})^{\top}$. Together with $g_{0}^{\top}=g_{0}$ and $\boldsymbol{U}=\left(\boldsymbol{V} g_{0}\right)^{\top}$, this shows that $g$ given by (3.11) is symmetric.

Remark 3.5. Lemma 3.2 and Proposition 3.2 also hold with transposition replaced by any involutory anti-automorphism of the matrix algebra, hence in particular for Hermitian conjugation. The Hermitian reduction of the non-autonomous chiral model appears in particular in the context of the (electro-vacuum) Einstein-Maxwell equations in four dimensions with two commuting Killing vector fields (also see [15]). 
In terms of the matrix

$$
\boldsymbol{\Gamma}:=-\boldsymbol{Q} \tilde{\boldsymbol{X}}=\epsilon\left(\tilde{\boldsymbol{P}}^{\top}\right)^{-1} \tilde{\boldsymbol{X}}
$$

which is symmetric under the assumptions of Proposition 3.2 (see the proof of Proposition 3.2), the Sylvester equation (3.10) takes the form of a Stein equation,

$$
\boldsymbol{\Gamma}+\epsilon \tilde{\boldsymbol{P}}^{\top} \boldsymbol{\Gamma} \tilde{\boldsymbol{P}}=\boldsymbol{V} g_{0} \boldsymbol{V}^{\top}
$$

Implementing the assumptions of Proposition 3.2 in the solution formula (3.11), we have

$$
g=\left(I-g_{0} \boldsymbol{V}^{\top} \boldsymbol{\Gamma}^{-1} \boldsymbol{V}\right) g_{0}
$$

If $\tilde{\boldsymbol{P}}$ is diagonal, i.e., $\tilde{\boldsymbol{P}}=\operatorname{diag}\left(\tilde{p}_{1}, \ldots, \tilde{p}_{n}\right)$, and if $\tilde{p}_{i} \tilde{p}_{j} \neq-\epsilon$ for all $i, j$, then the solution of (3.16) is given (via Example 3.4) by

$$
\boldsymbol{\Gamma}_{i j}=\epsilon \frac{\left(\boldsymbol{V} g_{0} \boldsymbol{V}^{\top}\right)_{i j}}{\tilde{p}_{i} \tilde{p}_{j}+\epsilon} .
$$

This is essentially the corresponding matrix (usually denoted by $\Gamma$ ) in the Belinski-Zakharov method $[2,3]$.

Remark 3.6. From Remark 2.3 we deduce the following superposition result. Let $\left(\tilde{\boldsymbol{P}}_{i}, \boldsymbol{V}_{i}\right), i=$ $1, \ldots, N$, be solutions of (3.3) and (3.8) with $\tilde{\boldsymbol{Q}}_{i}=-\epsilon\left(\tilde{\boldsymbol{P}}_{i}^{\boldsymbol{\top}}\right)^{-1}$, for the same seed solution $g_{0}$. Then $\tilde{\boldsymbol{P}}=\operatorname{block}-\operatorname{diag}\left(\tilde{\boldsymbol{P}}_{1}, \ldots, \tilde{\boldsymbol{P}}_{N}\right)$ and $\boldsymbol{V}=\left(\boldsymbol{V}_{1}^{\boldsymbol{\top}}, \ldots, \boldsymbol{V}_{N}^{\boldsymbol{\top}}\right)^{\boldsymbol{\top}}$ solve $(3.3)$ and $(3.8)$ with $\tilde{\boldsymbol{Q}}=-\epsilon\left(\tilde{\boldsymbol{P}}^{\boldsymbol{\top}}\right)^{-1}$. If $\operatorname{spec}(\tilde{\boldsymbol{P}}) \cap \operatorname{spec}\left(-\epsilon\left(\tilde{\boldsymbol{P}}^{\boldsymbol{\top}}\right)^{-1}\right)=\varnothing$, and if (3.16) has an invertible solution $\boldsymbol{\Gamma}$, then (3.17) is again a symmetric solution of (1.1).

\section{Solutions of the vacuum Einstein equations}

In $D$ dimensions, let us consider a space-time metric of the form

$$
d s^{2}=g_{\alpha \beta} d x^{\alpha} d x^{\beta}+f\left(\epsilon d \rho^{2}+d z^{2}\right)
$$

where the (real) components $g_{\alpha \beta}, \alpha, \beta=1, \ldots, m$, and the function $f$ only depend on the coordinates $\rho$ and $z$ (and thus not on $x^{1}, \ldots, x^{m}$ ). The metric then obviously admits $m=D-2$ commuting Killing vector fields ${ }^{11}$. For the next result, see, e.g., $[2,5,25,73]^{12}$. If

(1) the matrix $g=\left(g_{\alpha \beta}\right)$ satisfies $\operatorname{det} g=-\epsilon \rho^{2}$,

(2) $g$ solves the $m \times m$ non-autonomous chiral model equation (1.1),

(3) $f$ is a solution of the compatible system of linear equations

$$
(\ln f)_{\rho}=-\frac{1}{\rho}+\frac{1}{4 \rho} \operatorname{tr}\left(\mathcal{U}^{2}-\epsilon \mathcal{V}^{2}\right), \quad(\ln f)_{z}=\frac{1}{2 \rho} \operatorname{tr}(\mathcal{U} \mathcal{V})
$$

where $\mathcal{U}:=\rho g_{\rho} g^{-1}$ and $\mathcal{V}:=\rho g_{z} g^{-1}$,

\footnotetext{
${ }^{11}$ It is not the most general metric admitting $m=D-2$ commuting Killing vector fields. See, e.g., [2].

${ }^{12}$ There are also reductions of the Einstein vacuum equations to the non-autonomous chiral model equation using a non-Abelian Lie algebra of Killing vector fields. See the case with a null (i.e., lightlike, or isotropic) Killing vector field in four dimensions treated in [67].
} 
then the metric is Ricci-flat, hence a solution of the vacuum Einstein equations (with vanishing cosmological constant).

Since $g$ has to be real and symmetric, corresponding conditions have to be imposed on the matrix data of the class of solutions obtained in Section 3, so that these solutions determine Ricciflat metrics (also see [15]). Such conditions have been found in Section 3.4, and, accordingly, in all examples of this section we shall set

$$
\tilde{\boldsymbol{Q}}=-\left(\tilde{\boldsymbol{P}}^{-1}\right)^{\top}, \quad \boldsymbol{U}=\left(\boldsymbol{V} g_{0}\right)^{\top}
$$

Typically we will regard these equations as defining $\tilde{\boldsymbol{Q}}$ and $\boldsymbol{U}$ in terms of $\tilde{\boldsymbol{P}}, \boldsymbol{V}$ and $g_{0}$. In most of the examples below, $\tilde{\boldsymbol{P}}$ is diagonal. The (symmetric) solution of the non-autonomous chiral model is then given by (3.17) with $\boldsymbol{\Gamma}$ in (3.18). If $\tilde{\boldsymbol{P}}$ is not diagonal, we have to proceed via the solution (3.13) of the Sylvester equation (still assuming that the spectrum condition holds) and (3.15).

According to the following remark, the determinant condition (1) can always be achieved if $m$ is odd. There is a slight restriction if $m$ is even.

Remark 4.1. By taking the trace of (1.1), one finds that det $g$ and any power of it is a solution of (1.1) in the scalar case (also see $[2,4]$ ). We further note that $\hat{g}=w g$, with any non-vanishing solution $w$ of the scalar equation, is again a solution of (1.1). For a solution $g$ given by (3.17), using (3.12) and (4.2) we find that

$$
w=\left(\frac{\rho^{2}}{(\operatorname{det} \tilde{\boldsymbol{P}})^{2}(-1)^{n+1} \epsilon \operatorname{det} g_{0}}\right)^{1 / m}
$$

achieves that $\operatorname{det} \hat{g}=-\epsilon \rho^{2}$. Since $w$ has to be real, for even $m$ this requires $(-1)^{n}\left(-\epsilon \operatorname{det} g_{0}\right)>0$.

In the next subsection, we address (4.1). Then we sketch a useful procedure to construct non-diagonal metrics from diagonal ones in such a way that the diagonal metric is recovered by setting some parameters to zero. A collection of relevant examples in four and five space-time dimensions follows. The method is indeed of most interest for $D=4$ and $D=5$. The higher the number of dimensions, the more restrictive is the assumption of $D-2$ commuting Killing vector fields for the set of solutions of the vacuum Einstein equations.

\subsection{Solutions of the equations for the metric function $f$}

Example 4.1. For a diagonal solution $g_{0}$ of (1.1), so that $\left(g_{0}\right)_{\alpha \alpha}$ solves (3.9), (4.1) leads to

$$
f_{0}=\kappa \rho^{-1} \prod_{\alpha=1}^{m} \mathfrak{f}_{\alpha}
$$

where $\kappa$ is an arbitrary constant and $\mathfrak{f}_{\alpha}$ has to be a solution of

$$
(\ln \mathfrak{f})_{\rho}=\frac{\rho}{4}\left((\ln w)_{\rho}^{2}-\epsilon(\ln w)_{z}^{2}\right), \quad(\ln \mathfrak{f})_{z}=\frac{\rho}{2}(\ln w)_{\rho}(\ln w)_{z},
$$

with $w$ replaced by $\left(g_{0}\right)_{\alpha \alpha}$. If $w$ is a constant, then also $\mathfrak{f}$. Let us write $\mathfrak{f}[w]$ for the solution of the above equations for a given solution $w$ of (3.9), and let $\propto$ denote equality up to a non-zero constant factor. In particular, we have

$$
\mathfrak{f}\left[\tilde{\mu}_{i}\right] \propto \frac{\tilde{\mu}_{i}}{\sqrt{\tilde{\mu}_{i}^{2}+\epsilon \rho^{2}}}, \quad \text { where } \quad \tilde{\mu}_{i}= \pm \sqrt{\left(z-a_{i}\right)^{2}+\epsilon \rho^{2}}-\left(z-a_{i}\right)
$$


(cf. (3.6)). More generally, we find

$$
\begin{aligned}
\mathfrak{f}\left[\rho^{k} \frac{\tilde{\mu}_{1} \cdots \tilde{\mu}_{r}}{\tilde{\mu}_{1}^{\prime} \cdots \tilde{\mu}_{s}^{\prime}}\right] \propto & \rho^{k^{2} / 4}\left(\frac{\tilde{\mu}_{1} \cdots \tilde{\mu}_{r}}{\tilde{\mu}_{1}^{\prime} \cdots \tilde{\mu}_{s}^{\prime}}\right)^{k / 2}\left(\prod_{i=1}^{r} \mathfrak{f}\left[\tilde{\mu}_{i}\right]\right)\left(\prod_{j=1}^{s} \mathfrak{f}\left[\tilde{\mu}_{j}^{\prime}\right]\right)\left(\prod_{i<k} \mathfrak{F}\left[\tilde{\mu}_{i}, \tilde{\mu}_{k}\right]\right) \\
& \times\left(\prod_{j<l} \mathfrak{F}\left[\tilde{\mu}_{j}^{\prime}, \tilde{\mu}_{l}^{\prime}\right]\right) \prod_{i}^{r} \prod_{j}^{s} \mathfrak{F}\left[\tilde{\mu}_{i}, \tilde{\mu}_{j}^{\prime}\right]^{-1},
\end{aligned}
$$

where $\tilde{\mu}_{i}^{\prime}$ is $\tilde{\mu}_{i}$ with the constant $a_{i}$ replaced by some constant $a_{i}^{\prime}$, and we introduced the abbreviation

$$
\mathfrak{F}\left[\tilde{\mu}_{i}, \tilde{\mu}_{j}\right]=\frac{\tilde{\mu}_{i} \tilde{\mu}_{j}}{\tilde{\mu}_{i} \tilde{\mu}_{j}+\epsilon \rho^{2}}=\frac{\tilde{\mu}_{i}-\tilde{\mu}_{j}}{2\left(a_{i}-a_{j}\right)},
$$

where the last equality holds if $a_{i} \neq a_{j}$. The $\tilde{\mu}_{i}$ (and also the $\tilde{\mu}_{i}^{\prime}$ ) need not be distinct in the above formula. Using these results in our formula for $f_{0}$, we have the solution of (4.1) for a large class of diagonal solutions of (1.1). See Appendix A for some details, and also [3].

For the proofs of the following propositions, see Appendix B.

Proposition 4.1. Let $f_{0}$ be a solution of (4.1) for a seed $g_{0}$ (solution of the Miura equation). Let $g$ be a corresponding solution of the non-autonomous chiral model from the family obtained in Section 3 (i.e., given by (3.11), where the ingredients are subject to the respective equations). Then

$$
f=\kappa f_{0} \rho^{-n} \frac{\operatorname{det} \tilde{\boldsymbol{P}} \operatorname{det} \tilde{\boldsymbol{Q}} \operatorname{det} \tilde{\boldsymbol{X}}}{\left[\operatorname{det}\left(\boldsymbol{I}+\epsilon \tilde{\boldsymbol{P}}^{2}\right) \operatorname{det}\left(\boldsymbol{I}+\epsilon \tilde{\boldsymbol{Q}}^{2}\right)\right]^{1 / 2}},
$$

with an arbitrary constant $\kappa$, solves (4.1) with the right hand sides evaluated with $g$.

Proposition 4.1 does neither assume that $g_{0}$ and $g$ are symmetric, nor the reduction conditions (4.2). The following corollary now specializes to the case of this reduction.

Corollary 4.1. Let $g_{0}$ be symmetric, $\tilde{\boldsymbol{Q}}^{\top}=-\epsilon \tilde{\boldsymbol{P}}^{-1}, \operatorname{spec}(\tilde{\boldsymbol{P}}) \cap \operatorname{spec}(\tilde{\boldsymbol{Q}})=\varnothing$, and $\boldsymbol{U}=\left(\boldsymbol{V} g_{0}\right)^{\top}$. Then the solution of (4.1) corresponding to $g$ given by (3.17) is

$$
f=\kappa f_{0} \rho^{-n} \frac{(\operatorname{det} \tilde{\boldsymbol{P}})^{2}}{\operatorname{det}\left(\boldsymbol{I}+\epsilon \tilde{\boldsymbol{P}}^{2}\right)} \operatorname{det} \boldsymbol{\Gamma},
$$

with an arbitrary constant $\kappa$.

A convenient formula for the determinant of the solution $\boldsymbol{\Gamma}$ of the Stein equation seems not to be available in the literature.

If we have to modify a solution $g$ in order to achieve the determinant condition (1), the following result is of great help.

Proposition 4.2. Let $f$ be a solution of (4.1) for a given solution $g$ from the class obtained in Section 3, and let $f_{(w)}$ be a solution of (4.1) for a scalar solution $w$ of the non-autonomous chiral model (1.1). Then

$$
\hat{f}=\left(\rho f_{(w)}\right)^{m-1} \frac{f_{(w \operatorname{det} g)}}{f_{(\operatorname{det} g)}} f
$$

solves (4.1) with $\hat{g}=w g$ (which also solves (1.1) according to Remark 4.1). If $\operatorname{det} \hat{g}=-\epsilon \rho^{2}$, this reduces to

$$
\hat{f}=\frac{w f}{\left(\rho f_{(w)}\right)^{m}} .
$$


Due to these results, the problem of explicitly computing solutions of the vacuum Einstein equations, based on the results in Section 3 and with a diagonal seed, essentially boils down to that of solving the linear equations (3.8) for $\boldsymbol{V}$. For diagonal $\tilde{\boldsymbol{Q}}=-\epsilon \tilde{\boldsymbol{P}}^{-1}$, solutions of the latter have already been obtained in Example 3.3. Although we do not yet have general results in case of a non-diagonal $\tilde{\boldsymbol{Q}}$, corresponding examples are treated below in Examples 4.3 and 4.4.

\subsection{From diagonal to non-diagonal solutions}

In the following, we impose the reduction conditions (4.2), which determine $\tilde{\boldsymbol{Q}}$ in terms of $\tilde{\boldsymbol{P}}$, and $\boldsymbol{U}$ in terms of $\boldsymbol{V}$ and $g_{0}$, and restrict our considerations to the case $\epsilon=1$.

We translate a procedure due to Pomeransky [60], which has been formulated and applied in the Belinski-Zakharov (BZ) approach, to our framework. We start with a diagonal (hence in particular static) solution of the above dimensionally reduced vacuum Einstein equations. The corresponding solution $\tilde{g}$ of (1.1) is thus diagonal. According to Remark 2.2 we may still simplify it by multiplication with a suitable scalar solution of (1.1). Now some (anti-) solitons are removed from it in the following way.

- Removal of a soliton $p_{i}=\rho \mu_{i}^{-1}$ (i.e., a soliton at $z=a_{i}$ ) from $\tilde{g}_{\alpha \alpha}$. This means multiplication of $\tilde{g}_{\alpha \alpha}$ by $-p_{i}^{-2}$. In the BZ language, the corresponding trivial BZ vector has a 1 at the $\alpha$ th position and otherwise zeros.

- Removal of an anti-soliton $\bar{p}_{j}=-p_{j}^{-1}=-\rho^{-1} \mu_{j}$ (i.e., an anti-soliton at $z=a_{j}$ ) from $\tilde{g}_{\beta \beta}$. This means multiplication of $\tilde{g}_{\beta \beta}$ by $\bar{p}_{j}^{-2}=-p_{j}^{2}$.

Afterwards these solitons are reintroduced, with more freedom, in the following way.

- In order to reintroduce the corresponding soliton, set $\tilde{\boldsymbol{P}}_{k k}=p_{i}$, for some $k$. The $k$ th row of the matrix $\boldsymbol{V}$ that solves (3.8) (with $\tilde{\boldsymbol{Q}}^{\top}=-\tilde{\boldsymbol{P}}^{-1}$ ), or rather the vector formed by its constant coefficients, generalizes the aforementioned trivial BZ vector. It's $\alpha$ th component has to be non-zero.

- To reintroduce the corresponding anti-soliton, set $\tilde{\boldsymbol{P}}_{l l}=\bar{p}_{j}$, for some $l$. The $l$ th row of the matrix $\boldsymbol{V}$ generalizes the corresponding trivial BZ vector if its $\beta$ th component is non-zero.

After multiplication with a suitable scalar solution of (1.1), in order to achieve the determinant condition, this leads to a non-diagonal (and thus typically stationary) generalization of the original diagonal metric. We recover the latter by suitably fixing the parameters introduced in the second step.

In particular, this offers the possibility to reconstruct a known non-diagonal solution from its diagonal specialization (provided the latter can be achieved) via the solution-generating technique. Some well-known examples of four-dimensional space-times are recovered in Section 4.3 in this way. The above procedure will be applied in the five-dimensional case in Section 4.4.

Remark 4.2. According to an observation at the end of Remark 2.2, any transformation $\boldsymbol{V} \mapsto$ $\boldsymbol{W} \boldsymbol{V}$ with a constant $n \times n$ matrix $\boldsymbol{W}$ that commutes with $\tilde{\boldsymbol{P}}^{\top}$ (i.e., $\left[\boldsymbol{W}, \tilde{\boldsymbol{P}}^{\top}\right]=0$ ) leaves $g$ given by (3.17) invariant. If $\tilde{\boldsymbol{P}}$ is diagonal, any diagonal $\boldsymbol{W}$ commutes with it. We can then use such a transformation of $\boldsymbol{V}$ to scale in each row of $\boldsymbol{V}$ one of the non-zero constant coefficients to a fixed value like 1 . In the above step of reintroduction of solitons, without restriction of generality we can thus fix the constants appearing in the respective positions of the rows of $\boldsymbol{V}$. If $\tilde{\boldsymbol{P}}=\tilde{\boldsymbol{P}}_{r}$ (see Example 3.2), then $\boldsymbol{W}$ is an arbitrary constant, lower-triangular Toeplitz matrix and we can again rescale one non-zero coefficient in each row of the corresponding matrix $\boldsymbol{V}$ to a chosen value (different from zero), without changing the corresponding solution $g$. This feature generalizes to a $\tilde{\boldsymbol{P}}$ that is block-diagonally composed of matrices $\tilde{\boldsymbol{P}}_{r}$. 


\subsection{Solutions of the vacuum Einstein equations in four dimensions}

Let $D=4$, hence $m=2$, and $\epsilon=1$. We thus consider stationary and axially symmetric solutions of the vacuum Einstein equations. The metric components $g_{\alpha \beta}, \alpha, \beta=1,2$, should then refer to coordinates $x^{1}=t$ (time) and $x^{2}=\varphi$ (angle around the symmetry $z$-axis), $\rho$ is the coordinate distance from the axis. This interpretation in general imposes additional conditions on the metric. The simple solution

$$
g_{0}=\left(\begin{array}{cc}
-1 & 0 \\
0 & \rho^{2}
\end{array}\right)
$$

of (1.1) corresponds to the four-dimensional Minkowski metric $d s^{2}=-d t^{2}+\rho^{2} d \varphi^{2}+d \rho^{2}+d z^{2}$ in cylindrical coordinates. The solution $f_{0}$ of (4.1) with $g$ given by (4.6) is simply a constant, which can be set to 1 (since this can be achieved by a coordinate transformation).

In order to facilitate comparison with the relevant literature, in the following we will mainly use the $\mu_{i}$ introduced in (3.6), instead of the $p_{i}$, see Remark 3.1. Furthermore, we will frequently use the abbreviation (also see [73])

$$
\mathcal{R}_{i j}=\rho^{2}+\mu_{i} \mu_{j}=2\left(a_{i}-a_{j}\right) \frac{\mu_{i} \mu_{j}}{\mu_{i}-\mu_{j}},
$$

where the last equality holds if $a_{i} \neq a_{j}$.

Example 4.2 (Kerr-NUT). In order to recover the Kerr-NUT metric, we start with its static specialization, the Schwarzschild metric. The latter corresponds to the following solution of (1.1),

$$
\tilde{g}=\left(\begin{array}{cc}
-\frac{\mu_{2}}{\mu_{1}} & 0 \\
0 & \rho^{2} \frac{\mu_{1}}{\mu_{2}}
\end{array}\right) .
$$

Next we remove a soliton at $z=a_{1}$ from $\tilde{g}_{11}$ and a soliton at $z=a_{2}$ from $\tilde{g}_{22}$. The resulting matrix is simplified by rescaling it with the inverse of

$$
w=\rho^{-2} \mu_{1} \mu_{2} .
$$

We obtain the seed solution

$$
g_{0}=w^{-1} \tilde{g} \operatorname{diag}\left(-\left(\rho / \mu_{1}\right)^{-2},-\left(\rho / \mu_{2}\right)^{-2}\right),
$$

which is nothing but (4.6). Next we reintroduce the two solitons $(n=2)$ via

$$
\tilde{\boldsymbol{P}}=\operatorname{diag}\left(\frac{\rho}{\mu_{1}}, \frac{\rho}{\mu_{2}}\right) \text {. }
$$

The respective solution of (3.8) (with $\tilde{\boldsymbol{Q}}=-\tilde{\boldsymbol{P}}^{-1}$ ) is

$$
\boldsymbol{V}=\left(\begin{array}{ll}
v_{11} & v_{12} \mu_{1}^{-1} \\
v_{21} & v_{22} \mu_{2}^{-1}
\end{array}\right)
$$

with constants $v_{i \alpha}$ (see Example 3.3). According to Remark 4.2, we can set $v_{11}=v_{22}=1$ without restriction of generality. Let us rename the remaining parameters in $\boldsymbol{V}$,

$$
c_{1}=v_{12}, \quad c_{2}=v_{21} .
$$


Now we obtain the matrix $\boldsymbol{\Gamma}$ from (3.18). Then $\hat{g}=w g$, with $g$ given by (3.17), satisfies the determinant condition, i.e., $\operatorname{det} \hat{g}=-\rho^{2}$, and has the components

$$
\begin{aligned}
& \hat{g}_{11}=\frac{1}{h}\left(\left(c_{1}^{2}+c_{2}^{2}\right) \rho^{2} \mu_{1} \mu_{2}\left(\mu_{1}-\mu_{2}\right)^{2}-\mu_{1} \mu_{2}\left(\left(1+c_{1}^{2} c_{2}^{2}\right) \mathcal{R}_{12}^{2}-2 c_{1} c_{2} \mathcal{R}_{11} \mathcal{R}_{22}\right)\right), \\
& \hat{g}_{12}=\frac{\left(\mu_{1}-\mu_{2}\right) \mathcal{R}_{12}}{h}\left(c_{1}\left(c_{2}^{2} \mu_{2}^{2}-\rho^{2}\right) \mathcal{R}_{11}+c_{2}\left(c_{1}^{2} \rho^{2}-\mu_{1}^{2}\right) \mathcal{R}_{22}\right), \\
& \hat{g}_{22}=\frac{1}{h}\left(-\frac{\left(\mu_{1}-\mu_{2}\right)^{2}}{\mu_{1} \mu_{2}}\left(c_{1}^{2} \rho^{8}+c_{2}^{2} \mu_{1}^{4} \mu_{2}^{4}\right)+\frac{\rho^{2}}{\mu_{1} \mu_{2}}\left(\left(\mu_{1}^{4}+c_{1}^{2} c_{2}^{2} \mu_{2}^{4}\right) \mathcal{R}_{12}^{2}-2 c_{1} c_{2} \mu_{1}^{2} \mu_{2}^{2} \mathcal{R}_{11} \mathcal{R}_{22}\right)\right),
\end{aligned}
$$

where

$$
h=\left(\mu_{1}-\mu_{2}\right)^{2}\left(c_{1}^{2} \rho^{4}+c_{2}^{2} \mu_{1}^{2} \mu_{2}^{2}\right)+\left(\mu_{1}^{2}+c_{1}^{2} c_{2}^{2} \mu_{2}^{2}\right) \mathcal{R}_{12}^{2}-2 c_{1} c_{2} \mu_{1} \mu_{2} \mathcal{R}_{11} \mathcal{R}_{22} .
$$

Furthermore, according to Example 4.1, we have

$$
f_{(w)} \propto \frac{\mu_{1} \mu_{2}}{\sqrt{\mathcal{R}_{11} \mathcal{R}_{22}} \mathcal{R}_{12}} .
$$

Corollary 4.1 and Proposition 4.2 then yield

$$
\hat{f}=-\frac{\kappa}{\mu_{1} \mu_{2} \mathcal{R}_{11} \mathcal{R}_{22}} h,
$$

with a constant $\kappa$. Setting $a_{2}=-a_{1}=\sigma$,

$$
c_{1}=\frac{\mathfrak{m}+\sigma}{\mathfrak{a}+\mathfrak{b}}, \quad c_{2}=\frac{\mathfrak{a}+\mathfrak{b}}{\mathfrak{m}-\sigma}, \quad \kappa=\mathcal{C} \frac{(\mathfrak{m}-\sigma)^{2}}{4 \sigma^{2}}, \quad \sigma=\sqrt{\mathfrak{m}^{2}-\mathfrak{a}^{2}+\mathfrak{b}^{2}},
$$

with new constants $\mathfrak{a}, \mathfrak{b}, \mathfrak{m}$ and $\mathcal{C}$, and passing over from the coordinates $\rho$ and $z$ to new coordinates $r$ and $\theta$ via

$$
\rho=\sqrt{(r-\mathfrak{m})^{2}-\sigma^{2}} \sin \theta, \quad z=(r-\mathfrak{m}) \cos \theta,
$$

we obtain the Kerr-NUT metric in Boyer-Lindquist coordinates, as given by (8.48) and (8.49) in [2]. We can compose $N$ Kerr-NUT data blockwise into larger matrices (the new $\tilde{\boldsymbol{P}}$ and $\boldsymbol{V}$ are then $2 N \times 2 N$, respectively $2 N \times 2$ matrices), and obtain again a symmetric solution of the non-autonomous chiral model equation, see Remark 3.6. The determinant condition is again achieved by multiplication with a suitable scalar. In this way we can recover the multi-Kerr-NUT solutions [50].

Choosing for $\tilde{\boldsymbol{P}}$ the non-diagonal solution of (3.4) resulting from an $n \times n$ Jordan block matrix $\boldsymbol{A}_{n}$ (see Example 3.2), we should expect that the resulting family of solutions of the non-autonomous chiral model can be obtained alternatively via a "soliton coincidence" limit ("pole fusion" in the Belinski-Zakharov formalism [2]) of the family of solutions obtained with a diagonal $\tilde{\boldsymbol{P}}$ (with distinct eigenvalues). This is confirmed by the following example.

Example 4.3. Again, let $n=2$ and $g_{0}$ as in (4.6). Now we choose

$$
\tilde{\boldsymbol{P}}=\frac{\rho}{\mu_{1}}\left(\begin{array}{cc}
1 & -\mathfrak{R}^{-1} \\
0 & 1
\end{array}\right),
$$

where $\mu_{1}=\mathfrak{R}-z$ with $\mathfrak{R}=\sqrt{z^{2}+\rho^{2}}$. This is $\tilde{\boldsymbol{P}}_{2}$ in Example 3.2 (with $a=0$ ). We find the solution

$$
\boldsymbol{V}=\left(\begin{array}{cc}
v_{11} & v_{12} \mu_{1}^{-1} \\
v_{21} & v_{22} \mu_{1}^{-1}-2 v_{12} \mathcal{R}_{11}^{-1}
\end{array}\right)
$$


of (3.8), with constants $v_{i \alpha}$. Computing the solution of the Sylvester equation via (3.13), the symmetric matrix $\boldsymbol{\Gamma}$ is obtained from (3.15). We have to rescale the resulting $g$, given by (3.17), to $\hat{g}=w g$ with $w=\rho^{-2} \mu_{1}^{2}$, in order to arrange the determinant condition. It turns out that $\hat{g}$ only depends on the parameters $v_{i \alpha}$ via $\alpha=v_{12} / v_{11}$ and $\beta=\operatorname{det}\left(v_{i \alpha}\right) / v_{11}^{2}$. Furthermore, we find that $\hat{g}$ also results from the corresponding (Kerr-NUT) solution in Example 4.2 in the limit $\sigma \rightarrow 0$, after setting $c_{1}=\alpha$ and $c_{2}=\alpha^{-2}(\alpha-2 \beta \sigma)$.

The Kerr-NUT metric consists of two solitons of multiplicity one. The Tomimatsu-Sato metrics [72] are known to generalize it to two solitons of multiplicity $\delta \geq 1$ (see, e.g., [2] ${ }^{13}$. We should then expect that, for fixed $\delta$, this solution can be obtained alternatively with $\tilde{\boldsymbol{P}}$ consisting of two blocks $\tilde{\boldsymbol{P}}_{\delta}$ as given in Example 3.2 (corresponding to the $\delta \times \delta$ Jordan block $\boldsymbol{A}_{\delta}$ ). For $\delta=2$ this is confirmed in the next example.

Example 4.4 (Tomimatsu-Sato). In the static limit, the $\delta=2$ Tomimatsu-Sato metric (also see [49]) reduces to the Zipoy-Voorhees metric. The latter corresponds to the diagonal solution

$$
\tilde{g}=\operatorname{diag}\left(-\mu_{1}^{-2} \mu_{2}^{2}, \rho^{2} \mu_{1}^{2} \mu_{2}^{-2}\right)
$$

of (1.1). Now we remove twice a soliton at $z=a_{1}=\sigma$ from $\tilde{g}_{11}$, and also twice a soliton at $z=a_{2}=-\sigma$ from $\tilde{g}_{22}$. With a simplifying scaling we obtain the seed

$$
g_{0}=w^{-1} \tilde{g} \operatorname{diag}\left(\rho^{-4} \mu_{1}^{4}, \rho^{-4} \mu_{2}^{4}\right), \quad w=\rho^{-4} \mu_{1}^{2} \mu_{2}^{2} .
$$

The resulting $g_{0}$ is again (4.6). Next we reintroduce the two double-solitons via ${ }^{14}$

$$
\tilde{\boldsymbol{P}}=\left(\begin{array}{cccc}
\rho \mu_{1}^{-1} & -2 \rho \mathcal{R}_{11}^{-1} & 0 & 0 \\
0 & \rho \mu_{1}^{-1} & 0 & 0 \\
0 & 0 & \rho \mu_{2}^{-1} & -2 \rho \mathcal{R}_{22}^{-1} \\
0 & 0 & 0 & \rho \mu_{2}^{-1}
\end{array}\right)
$$

The solution of (3.8) is then given by

$$
\boldsymbol{V}=\left(\begin{array}{cc}
v_{11} & v_{12} \mu_{1}^{-1} \\
v_{21} & v_{22} \mu_{1}^{-1}-2 v_{12} \mathcal{R}_{11}^{-1} \\
v_{31} & v_{32} \mu_{2}^{-1} \\
v_{41} & v_{42} \mu_{2}^{-1}-2 v_{32} \mathcal{R}_{22}^{-1}
\end{array}\right)
$$

Again, the matrix $\boldsymbol{\Gamma}$ is obtained via (3.13) and (3.15). Next we have to compute $\hat{g}=w g$, with $g$ given by (3.17), and

$$
\hat{f}=\kappa \rho^{-6} w f_{(w)}^{-2} \frac{(\operatorname{det} \tilde{\boldsymbol{P}})^{2} \operatorname{det} \boldsymbol{\Gamma}}{\operatorname{det}\left(\boldsymbol{I}+\tilde{\boldsymbol{P}}^{2}\right)}, \quad \text { where } \quad f_{(w)}=\rho^{3}\left(\frac{\mu_{1}^{2} \mu_{2}^{2}}{\mathcal{R}_{11} \mathcal{R}_{12} \mathcal{R}_{22}}\right)^{2} .
$$

According to Remark 4.2, without restriction of generality we can set

$$
v_{11}=v_{21}=v_{32}=v_{42}=1 \text {. }
$$

Choosing

$$
v_{12}=v_{22}=v_{31}=v_{41}=\frac{\mathfrak{q}}{1+\mathfrak{p}}, \quad \kappa=\sigma^{-2}\left(\frac{1+\mathfrak{p}}{4 \mathfrak{p}}\right)^{4},
$$

\footnotetext{
${ }^{13}$ The Tomimatsu-Sato metrics are in fact limiting cases of multiple Kerr-NUT solutions [21,50].

${ }^{14}$ Noting that $p_{1} / \mathfrak{R}_{1}=2 \rho / \mathcal{R}_{11}$, this consists of two blocks of the form $\tilde{\boldsymbol{P}}_{2}$ in Example 3.2 .
} 
in terms of prolate spheroidal coordinates $x, y$, given by

$$
\rho=\sigma \sqrt{\left(x^{2}-1\right)\left(1-y^{2}\right)}, \quad z=\sigma x y,
$$

and after a coordinate transformation $t \mapsto t-4 \sigma \mathfrak{p}^{-1} \mathfrak{q} \varphi$, we obtain the $\delta=2$ Tomimatsu-Sato metric

$$
\begin{aligned}
d s^{2}= & -\frac{A}{B} d t^{2}+8 \sigma\left(1-y^{2}\right) \frac{\mathfrak{q} C}{\mathfrak{p} B} d t d \varphi+\sigma^{2}\left(1-y^{2}\right) \frac{D}{\mathfrak{p}^{2} B} d \varphi^{2} \\
& +\frac{B}{\mathfrak{p}^{4}\left(x^{2}-y^{2}\right)^{3}}\left(\frac{d x^{2}}{x^{2}-1}+\frac{d y^{2}}{1-y^{2}}\right)
\end{aligned}
$$

with

$$
\begin{aligned}
A= & \left(1-y^{2}\right)^{4} \mathfrak{g}(Z) \mathfrak{g}(-Z), \\
B= & \left(\mathfrak{g}(x)+\mathfrak{q}^{2} y^{4}\right)^{2}+4 \mathfrak{q}^{2} y^{2}\left(\mathfrak{p} x^{3}+1-(\mathfrak{p} x+1) y^{2}\right)^{2}, \\
C= & \mathfrak{q}^{2}(\mathfrak{p} x+1) y^{4}\left(-y^{2}+3\right)+\left(-2 \mathfrak{q}^{2}\left(\mathfrak{p} x^{3}+1\right)+(\mathfrak{p} x+1) \mathfrak{g}(x)\right) y^{2}-\left(2 \mathfrak{p} x^{3}-\mathfrak{p} x+1\right) \mathfrak{g}(x), \\
D= & \mathfrak{p}^{2} \mathfrak{q}^{4}\left(x^{2}-1\right) y^{8}+4 \mathfrak{q}^{2}(\mathfrak{p} x+1)\left(\mathfrak{p}^{3} x^{3}+3 \mathfrak{p}^{2} x^{2}-\mathfrak{p}^{3} x+4 \mathfrak{p} x-3 \mathfrak{p}^{2}+4\right) y^{6} \\
& -2 \mathfrak{q}^{2}\left(3 \mathfrak{p}^{4} x^{6}+4 \mathfrak{p}^{3} x^{5}-3 \mathfrak{p}^{4} x^{4}+8 \mathfrak{p}^{3} x^{3}+37 \mathfrak{p}^{2} x^{2}-12 \mathfrak{p}^{3} x+48 \mathfrak{p} x-13 \mathfrak{p}^{2}+24\right) y^{4} \\
& +4 \mathfrak{q}^{2}\left(\mathfrak{p}^{4} x^{8}-\mathfrak{p}^{4} x^{6}+4 \mathfrak{p}^{3} x^{5}-4 \mathfrak{p}^{3} x^{3}+15 \mathfrak{p}^{2} x^{2}+24 \mathfrak{p} x-3 \mathfrak{p}^{2}+12\right) y^{2} \\
& +\mathfrak{g}(x)\left(\mathfrak{p}^{4} x^{6}+6 \mathfrak{p}^{3} x^{5}-\mathfrak{p}^{4} x^{4}+16 \mathfrak{p}^{2} x^{4}-12 \mathfrak{p}^{3} x^{3}+32 \mathfrak{p} x^{3}+15 \mathfrak{p}^{2} x^{2}+6 \mathfrak{p}^{3} x-15 \mathfrak{p}^{2}+16\right),
\end{aligned}
$$

where $\mathfrak{p}^{2}+\mathfrak{q}^{2}=1$ and

$$
\mathfrak{g}(x)=\mathfrak{p}^{2} x^{4}+2 \mathfrak{p} x^{3}-2 \mathfrak{p} x-1, \quad Z^{2}=\frac{x^{2}-y^{2}}{1-y^{2}} .
$$

These are the expressions $(32)-(35)$ in $[49]^{15}$.

Remark 4.3. The $m=2$ non-autonomous chiral model is also related to the $D=4$ vacuum Einstein equations with two commuting Killing vector fields in another way, via the Ernst equation (see, e.g., [68]). In this case, one has to impose a different determinant condition: $\operatorname{det} g=1$. This allows a constant seed $g_{0}$, and thus constant matrices $\boldsymbol{U}, \boldsymbol{V}$, in which case the application of Theorem 2.1 is considerably simplified. The solutions obtained in this way include the multi-Kerr-NUT metrics (see [15] for details) ${ }^{16}$. A similar construction with $m=3$ leads to the (electrically and magnetically) charged generalizations, the multi-Demianski-Newman metrics [15].

\subsection{Solutions of the vacuum Einstein equations in five dimensions}

Let $m=3$ and $\epsilon=1$. In this case it seems that no relevant solutions can be obtained by choosing as the seed a diagonal solution of the non-autonomous chiral model corresponding to five-dimensional Minkowski space-time. It then becomes a subtle problem to choose a suitable seed solution. Here insights about the "rod structure" of the putative axis $\rho=0$ are of great help $[2,9,27,36,60]$. Some important solutions that have been obtained or recovered previously in the Belinski-Zakharov approach will now be presented in our framework. We use the procedure outlined in Section 4.2.

\footnotetext{
${ }^{15}$ We obtained a factor $\mathfrak{q}^{4}$ in the expression for $D$, whereas there is a factor $\mathfrak{q}^{2}$ in [49]. We believe that the latter is a typo.

${ }^{16}$ In $[78,80]$, solutions of the five-dimensional Einstein-Maxwell equations are constructed from a pair of solutions of the $m=2$ non-autonomous chiral model with symmetric $g$ and $\operatorname{det} g=1$.
} 


\subsubsection{Myers-Perry black holes}

The higher-dimensional generalization of a static black hole is given by the SchwarzschildTangherlini solution [71]. The corresponding diagonal solution of (1.1) is

$$
\tilde{g}=\operatorname{diag}\left(-\frac{\mu_{1}}{\mu_{2}}, \mu_{2}, \frac{\rho^{2}}{\mu_{1}}\right) .
$$

The seed $g_{0}$ is obtained from $\tilde{g}$ as follows:

- Remove a soliton at $z=a_{1}$ from $\tilde{g}_{33}$.

- Remove an anti-soliton at $z=a_{2}$ from $\tilde{g}_{22}$.

- Multiply the resulting matrix by $w^{-1}$, with $w=-\mu_{1} / \mu_{2}$, to achieve a simpler form.

This results in

$$
g_{0}=\operatorname{diag}\left(1, \frac{\rho^{2}}{\mu_{1}}, \mu_{2}\right) \text {. }
$$

According to Example 4.1, the associated solution of (4.1) is (up to a constant factor)

$$
f_{0}=\frac{\mu_{2}}{\sqrt{\mathcal{R}_{11} \mathcal{R}_{22}}}
$$

Next we reintroduce the soliton and the anti-soliton via

$$
\tilde{\boldsymbol{P}}=\operatorname{diag}\left(\frac{\rho}{\mu_{1}},-\frac{\mu_{2}}{\rho}\right) .
$$

The first row of the $2 \times 3$ matrix $\boldsymbol{V}$ should have a non-zero entry in the third component, which means we recreate the soliton at $z=a_{1}$ in the respective diagonal component of the seed. The second row of $\boldsymbol{V}$ should have a non-zero entry in the second component, which means we recreate the anti-soliton at $z=a_{2}$. According to Example 3.3, the solution of (3.8) (with $\tilde{\boldsymbol{Q}}=-\tilde{\boldsymbol{P}}^{-1}$ ) is given by

$$
\boldsymbol{V}=\left(\begin{array}{lll}
v_{11} & v_{12} \frac{\mu_{1}}{\mathcal{R}_{11}} & v_{13} \frac{\mathcal{R}_{12}}{\mu_{1} \mu_{2}} \\
v_{21} & v_{22} \frac{\mathcal{R}_{12}}{\rho^{2}} & v_{23} \frac{\mu_{2}}{\mathcal{R}_{22}}
\end{array}\right),
$$

with constants $v_{i \alpha}$. The corresponding solution of the Stein equation (3.16) is obtained from (3.18). With $g$ given by (3.17), the new solution

$$
\hat{g}=w g
$$

satisfies the determinant condition: $\operatorname{det} \hat{g}=-\rho^{2}$. We find

$$
f_{(w)}=\frac{\mathcal{R}_{12}}{\rho \sqrt{\mathcal{R}_{11} \mathcal{R}_{22}}}
$$

(up to a constant factor). Without restriction of generality, we can set $v_{13}=v_{22}=1$ (see Remark 4.2). With the restrictions and renamings

$$
v_{12}=v_{23}=0, \quad c_{1}=v_{11}, \quad c_{2}=v_{21},
$$


use of Corollary 4.1 and Proposition 4.2 yields

$$
\hat{f}=\kappa \mu_{2} \frac{c_{1}^{2} c_{2}^{2} \mu_{1}^{3} \mu_{2}-\left(\mu_{1}-\mu_{2}\right)^{2}\left(\mu_{1}\left(c_{1}^{2} \mu_{1} \mu_{2}+c_{2}^{2} \rho^{2}\right)+\mathcal{R}_{12}^{2}\right)}{\left(\mu_{1}-\mu_{2}\right)^{2} \mathcal{R}_{11} \mathcal{R}_{12} \mathcal{R}_{22}},
$$

with a constant $\kappa$. The corresponding metric is given by

$$
d s^{2}=\hat{g}_{11} d t^{2}+\hat{g}_{22} d \varphi^{2}+\hat{g}_{33} d \psi^{2}+2 \hat{g}_{12} d t d \varphi+2 \hat{g}_{13} d t d \psi+2 \hat{g}_{23} d \varphi d \psi+\hat{f}\left(d \rho^{2}+d z^{2}\right) .
$$

Setting

$$
a_{1}=-\sigma, \quad a_{2}=\sigma,
$$

with a constant $\sigma$, in terms of prolate spheroidal coordinates $x, y$, given by (4.7), we have

$$
\begin{aligned}
& \hat{g}_{11}=-\left(4 \sigma x+\left(\mathfrak{a}_{1}^{2}-\mathfrak{a}_{2}^{2}\right) y-\rho_{0}^{2}\right) \omega^{-1}, \\
& \hat{g}_{12}=-\mathfrak{a}_{1} \rho_{0}^{2}(1-y) \omega^{-1}, \\
& \hat{g}_{13}=-\mathfrak{a}_{2} \rho_{0}^{2}(1+y) \omega^{-1}, \\
& \hat{g}_{22}=\frac{1-y}{4}\left(4 \sigma x+\mathfrak{a}_{1}^{2}-\mathfrak{a}_{2}^{2}+\rho_{0}^{2}+2 \mathfrak{a}_{1}^{2} \rho_{0}^{2}(1-y) \omega^{-1}\right), \\
& \hat{g}_{23}=\frac{1}{2} \mathfrak{a}_{1} \mathfrak{a}_{2} \rho_{0}^{2}\left(1-y^{2}\right) \omega^{-1}, \\
& \hat{g}_{33}=\frac{1+y}{4}\left(4 \sigma x-\mathfrak{a}_{1}^{2}+\mathfrak{a}_{2}^{2}+\rho_{0}^{2}+2 \mathfrak{a}_{2}^{2} \rho_{0}^{2}(1+y) \omega^{-1}\right), \\
& \hat{f}=\frac{\omega}{8}, \quad \omega=4 \sigma x+\left(\mathfrak{a}_{1}^{2}-\mathfrak{a}_{2}^{2}\right) y+\rho_{0}^{2} .
\end{aligned}
$$

Performing a linear transformation of the coordinates $t, \varphi, \psi$,

$$
(t, \varphi, \psi) \mapsto\left(t-\mathfrak{a}_{1} \varphi-\mathfrak{a}_{2} \psi,-\frac{\mathfrak{a}_{1}}{c_{2}} \varphi+\frac{\mathfrak{a}_{2} c_{2}}{4 \sigma} \psi, \frac{\mathfrak{a}_{1} c_{1}}{4 \sigma} \varphi-\frac{\mathfrak{a}_{2}}{c_{1}} \psi\right)
$$

and setting

$$
\begin{array}{rlrl}
\sigma & =\frac{1}{4} \sqrt{\left(\rho_{0}^{2}-\mathfrak{a}_{1}^{2}-\mathfrak{a}_{2}^{2}\right)^{2}-4 \mathfrak{a}_{1}^{2} \mathfrak{a}_{2}^{2}}, & \kappa=\frac{\left(\mathfrak{a}_{1}^{2}-\mathfrak{a}_{2}^{2}\right)^{2}-\left(4 \sigma+\rho_{0}^{2}\right)^{2}}{16 \sigma \rho_{0}^{2}}, \\
c_{1}^{2}=\left|\frac{4 \sigma\left(\rho_{0}^{2}-\mathfrak{a}_{1}^{2}+\mathfrak{a}_{2}^{2}-4 \sigma\right)}{\left.\rho_{0}^{2}+\mathfrak{a}_{1}^{2}-\mathfrak{a}_{2}^{2}+4 \sigma\right)}\right|, & c_{2}^{2}=\left|\frac{4 \sigma\left(\rho_{0}^{2}+\mathfrak{a}_{1}^{2}-\mathfrak{a}_{2}^{2}-4 \sigma\right)}{\rho_{0}^{2}-\mathfrak{a}_{1}^{2}+\mathfrak{a}_{2}^{2}+4 \sigma}\right|,
\end{array}
$$

we recover the analogue of the Kerr metric, i.e., the Myers-Perry solution $[36,56,57]$ of the fivedimensional vacuum Einstein equations, precisely in the form of equation (19) in [60]. Switching on the constants $v_{12}$ and $v_{23}$ (which we set to zero above), leads to a more general class of space-times.

\subsubsection{Black saturn}

A black saturn [22] (also see $[11,69,79]$ ) is a black hole surrounded by a black ring ${ }^{17}$. In the following, we show how the black saturn solution, originally obtained in [22], can be recovered in our approach. Let us start with the same static solution of the non-autonomous chiral model as in [22],

$$
\tilde{g}=\operatorname{diag}\left(-\frac{\mu_{1} \mu_{3}}{\mu_{2} \mu_{4}}, \frac{\rho^{2} \mu_{4}}{\mu_{3} \mu_{5}}, \frac{\mu_{2} \mu_{5}}{\mu_{1}}\right) .
$$

This is motivated by a rod structure analysis (also see $[9,27,36]$ ). A suitable seed solution is then constructed as follows.

\footnotetext{
${ }^{17}$ Black rings are similar to black holes, but with horizon topology $S^{1} \times S^{D-3}$. They only appear in $D>4$ dimensions. See $[8,10,23,24,26,28,29,32,33,40,41,43,46,47,55,61,74-76]$.
} 
- Remove anti-solitons at $z=a_{1}$ and at $z=a_{3}$ from $\tilde{g}_{11}$.

- Remove a soliton at $z=a_{2}$ from $\tilde{g}_{11}$.

- Multiply the resulting matrix by $w^{-1}$ with $w=\frac{\rho^{2} \mu_{2}}{\mu_{1} \mu_{3}}$ to simplify its form.

This results in

$$
g_{0}=w^{-1} \tilde{g} \operatorname{diag}\left(-\frac{\rho^{2} \mu_{2}^{2}}{\mu_{1}^{2} \mu_{3}^{2}}, 1,1\right)=\operatorname{diag}\left(\frac{1}{\mu_{4}}, \frac{\mu_{1} \mu_{4}}{\mu_{2} \mu_{5}}, \frac{\mu_{3} \mu_{5}}{\rho^{2}}\right) .
$$

The corresponding solution of (4.1) is obtained via Example 4.1,

$$
f_{0}=k^{2} \mu_{3} \mu_{4} \mu_{5} \frac{\mathcal{R}_{12} \mathcal{R}_{15} \mathcal{R}_{24} \mathcal{R}_{45}}{\sqrt{\mathcal{R}_{11} \mathcal{R}_{22} \mathcal{R}_{33}} \mathcal{R}_{14} \mathcal{R}_{25} \mathcal{R}_{35} \mathcal{R}_{44} \mathcal{R}_{55}},
$$

with a constant $k$. We have $n=3$ and choose $\tilde{\boldsymbol{P}}$ diagonal with

$$
\tilde{\boldsymbol{P}}_{11}=-\mu_{1} / \rho, \quad \tilde{\boldsymbol{P}}_{22}=\rho / \mu_{2}, \quad \tilde{\boldsymbol{P}}_{33}=-\mu_{3} / \rho .
$$

The solution of the equations for $\boldsymbol{V}$ is then given by

$$
\boldsymbol{V}=\left(\begin{array}{ccc}
v_{11} \frac{\mathcal{R}_{14}}{\mu_{1}} & v_{12} \frac{\mathcal{R}_{12} \mathcal{R}_{15}}{\mathcal{R}_{11} \mathcal{R}_{14}} & v_{13} \frac{\rho^{2} \mu_{1}}{\mathcal{R}_{13} \mathcal{R}_{15}} \\
v_{21} \frac{\mu_{2} \mu_{4}}{\mathcal{R}_{24}} & v_{22} \frac{\mu_{2} \mu_{5} \mathcal{R}_{12} \mathcal{R}_{24}}{\mu_{1} \mu_{4} \mathcal{R}_{22} \mathcal{R}_{25}} & v_{23} \frac{\mu_{1} \mathcal{R}_{23} \mathcal{R}_{25}}{\mu_{2}^{2} \mu_{3} \mu_{5}} \\
v_{31} \frac{\mathcal{R}_{34}}{\mu_{3}} & v_{32} \frac{\mathcal{R}_{23} \mathcal{R}_{35}}{\mathcal{R}_{13} \mathcal{R}_{34}} & v_{33} \frac{\rho^{2} \mu_{3}}{\mathcal{R}_{33} \mathcal{R}_{35}}
\end{array}\right),
$$

with constants $v_{i \alpha}$. Without restriction of generality, we can set

$$
v_{11}=v_{21}=v_{31}=1 .
$$

The case considered in Section 2.2 of [22] should then correspond to the subclass of solutions given by

$$
v_{12}=v_{22}=v_{33}=0 .
$$

The authors of [22] then only elaborate the special case

$$
v_{32}=0
$$

further. In this case, however, the removal of the anti-soliton at $z=a_{3}$ from the original static metric and the subsequent reintroduction via $\tilde{\boldsymbol{P}}_{33}$ is actually redundant. This means that the black saturn space-time can already be obtained from $n=2$ data. The solution $\boldsymbol{\Gamma}$ of the Stein equation (3.16) is given by (3.18). With $g$ given by (3.17), we set $\hat{g}=w g$ to satisfy the determinant condition. Then $\hat{g}$ reduces to $\tilde{g}$ if $v_{13}=v_{23}=0$, as expected. We find

$$
f_{(w)}=\frac{\mathcal{R}_{12} \mathcal{R}_{23}}{\sqrt{\mathcal{R}_{11} \mathcal{R}_{22} \mathcal{R}_{33}} \mathcal{R}_{13}}
$$

(up to a constant factor) and, using Corollary 4.1 and Proposition 4.2,

$$
\hat{f}=-\kappa \rho^{-6} w f_{0} f_{(w)}^{-3} \frac{(\operatorname{det} \tilde{\boldsymbol{P}})^{2} \operatorname{det} \boldsymbol{\Gamma}}{\operatorname{det}\left(\boldsymbol{I}+\tilde{\boldsymbol{P}}^{2}\right)},
$$


which turns out to be a lengthy expression. Setting

$$
v_{13}=2 c_{1}\left(a_{1}-a_{5}\right), \quad v_{23}=c_{2}\left[4\left(a_{2}-a_{3}\right)\left(a_{2}-a_{4}\right)\right]^{-1}, \quad \kappa=4 \frac{\left(a_{1}-a_{2}\right)^{2}\left(a_{2}-a_{3}\right)^{2}}{\left(a_{1}-a_{3}\right)^{2}}
$$

with constants $c_{i}$, we obtain the metric

$$
d s^{2}=-\frac{H_{2}}{H_{1}}\left(d t+\frac{\omega}{H_{2}} d \psi\right)^{2}+H_{1}\left(k^{2} \mathcal{P}\left(d \rho^{2}+d z^{2}\right)+\frac{G_{1}}{H_{1}} d \varphi^{2}+\frac{G_{2}}{H_{2}} d \psi^{2}\right),
$$

where

$$
\begin{aligned}
G_{1} & =\frac{\rho^{2} \mu_{4}}{\mu_{3} \mu_{5}}, \quad G_{2}=\frac{\mu_{3} \mu_{5}}{\mu_{4}}, \quad \mathcal{P}=\mathcal{R}_{15} \mathcal{R}_{34}^{2} \mathcal{R}_{45} \\
H_{1} & =F^{-1}\left(M_{0}+c_{1}^{2} M_{1}+c_{2}^{2} M_{2}+c_{1} c_{2} M_{3}+c_{1}^{2} c_{2}^{2} M_{4}\right) \\
H_{2} & =\frac{\mu_{3}}{\mu_{4} F}\left(\frac{\mu_{1}}{\mu_{2}} M_{0}-c_{1}^{2} \frac{\rho^{2}}{\mu_{1} \mu_{2}} M_{1}-c_{2}^{2} \frac{\mu_{1} \mu_{2}}{\rho^{2}} M_{2}+c_{1} c_{2} M_{3}+c_{1}^{2} c_{2}^{2} \frac{\mu_{2}}{\mu_{1}} M_{4}\right), \\
F & =\mu_{1} \mu_{5}\left(\mu_{1}-\mu_{3}\right)^{2}\left(\mu_{2}-\mu_{4}\right)^{2}\left(\prod_{i=1}^{5} \mathcal{R}_{i i}\right) \mathcal{R}_{13} \mathcal{R}_{14} \mathcal{R}_{23} \mathcal{R}_{24} \mathcal{R}_{25} \mathcal{R}_{35},
\end{aligned}
$$

with

$$
\begin{aligned}
& M_{0}=\mu_{2} \mu_{5}^{2}\left(\mu_{1}-\mu_{3}\right)^{2}\left(\mu_{2}-\mu_{4}\right)^{2} \mathcal{R}_{12}^{2} \mathcal{R}_{14}^{2} \mathcal{R}_{23}^{2}, \\
& M_{1}=\rho^{2} \mu_{1}^{2} \mu_{2} \mu_{3} \mu_{4} \mu_{5}\left(\mu_{1}-\mu_{2}\right)^{2}\left(\mu_{1}-\mu_{5}\right)^{2}\left(\mu_{2}-\mu_{4}\right)^{2} \mathcal{R}_{23}^{2}, \\
& M_{2}=\rho^{2} \mu_{1}^{2} \mu_{2}^{-1} \mu_{3} \mu_{4} \mu_{5}\left(\mu_{1}-\mu_{2}\right)^{2}\left(\mu_{1}-\mu_{3}\right)^{2} \mathcal{R}_{14}^{2} \mathcal{R}_{25}^{2}, \\
& M_{3}=2 \mu_{1}^{2} \mu_{3} \mu_{4} \mu_{5}\left(\mu_{1}-\mu_{3}\right)\left(\mu_{1}-\mu_{5}\right)\left(\mu_{2}-\mu_{4}\right) \mathcal{R}_{11} \mathcal{R}_{22} \mathcal{R}_{14} \mathcal{R}_{23} \mathcal{R}_{25}, \\
& M_{4}=\mu_{1}^{4} \mu_{2}^{-1} \mu_{3}^{2} \mu_{4}^{2}\left(\mu_{1}-\mu_{5}\right)^{2} \mathcal{R}_{12}^{2} \mathcal{R}_{25}^{2}, \\
& \omega=\frac{2}{F \sqrt{G_{1}}}\left(c_{1} \Re_{1} \sqrt{M_{0} M_{1}}-c_{2} \mathfrak{R}_{2} \sqrt{M_{0} M_{2}}+c_{1}^{2} c_{2} \Re_{2} \sqrt{M_{1} M_{4}}-c_{1} c_{2}^{2} \mathfrak{R}_{1} \sqrt{M_{2} M_{4}}\right),
\end{aligned}
$$

and $\mathfrak{R}_{i}=\sqrt{\left(z-a_{i}\right)^{2}+\rho^{2}}$. This is the black saturn metric ${ }^{18}$ as given in [22,73], with some obvious changes in notation, but some deviations in the factors $\mu_{i}$ in the expressions for $M_{2}, M_{3}$ and $M_{4}$.

\subsubsection{Double Myers-Perry black hole solution}

In order to recover the double Myers-Perry black hole solution obtained in [39], we start with the matrix that determines a static two-black hole solution (cf. (3.1) in [70]),

$$
\tilde{g}=\operatorname{diag}\left(-\frac{\mu_{1} \mu_{4}}{\mu_{2} \mu_{5}}, \frac{\rho^{2} \mu_{3}}{\mu_{1} \mu_{4}}, \frac{\mu_{2} \mu_{5}}{\mu_{3}}\right) .
$$

Removal of two solitons $\mu_{2}, \mu_{5}$ and two anti-solitons $\mu_{1}, \mu_{4}$ from $\tilde{g}_{11}$, and simplification with a suitable factor, leads to

$$
g_{0}=w^{-1} \tilde{g} \operatorname{diag}\left(-\left(\frac{\mu_{1}^{2} \mu_{4}^{2}}{\mu_{2}^{2} \mu_{5}^{2}}\right)^{-1}, 1,1\right)=\operatorname{diag}\left(-1, \frac{\rho^{2} \mu_{3}}{\mu_{2} \mu_{5}}, \frac{\mu_{1} \mu_{4}}{\mu_{3}}\right), \quad w=\frac{\mu_{2} \mu_{5}}{\mu_{1} \mu_{4}} .
$$

\footnotetext{
${ }^{18}$ Additional conditions have to be imposed on the remaining parameters in order to achieve asymptotic flatness and absence of naked and conical singularities, see [11,22].
} 
According to Example 4.1, the corresponding solution of (4.1) is given by

$$
f_{0}=\frac{\mu_{1} \mu_{4}}{\mu_{3}}\left(\prod_{\substack{i=1 \\ i \neq 3}}^{5} \sqrt{\mathcal{R}_{i i}}\right)^{-1} \frac{\mathcal{R}_{13} \mathcal{R}_{23} \mathcal{R}_{34} \mathcal{R}_{35}}{\mathcal{R}_{14} \mathcal{R}_{25} \mathcal{R}_{33}} .
$$

We have $n=4$ and reintroduce the removed solitons and anti-solitons via

$$
\tilde{\boldsymbol{P}}=\operatorname{diag}\left(-\rho^{-1} \mu_{1},-\rho^{-1} \mu_{4}, \rho \mu_{2}^{-1}, \rho \mu_{5}^{-1}\right) .
$$

The solution of (3.8) is given by

$$
\boldsymbol{V}=\left(\begin{array}{ccc}
v_{11} & v_{12} \frac{\mathcal{R}_{12} \mathcal{R}_{15}}{\rho^{2} \mathcal{R}_{13}} & v_{13} \frac{\mu_{1} \mathcal{R}_{13}}{\mathcal{R}_{11} \mathcal{R}_{14}} \\
v_{21} & v_{22} \frac{\mathcal{R}_{24} \mathcal{R}_{45}}{\rho^{2} \mathcal{R}_{34}} & v_{23} \frac{\mu_{4} \mathcal{R}_{34}}{\mathcal{R}_{14} \mathcal{R}_{44}} \\
v_{31} & v_{32} \frac{\mu_{2} \mu_{5} \mathcal{R}_{23}}{\mu_{3} \mathcal{R}_{22} \mathcal{R}_{25}} & v_{33} \frac{\mu_{3} \mathcal{R}_{12} \mathcal{R}_{24}}{\mu_{1} \mu_{2} \mu_{4} \mathcal{R}_{23}} \\
v_{41} & v_{42} \frac{\mu_{2} \mu_{5} \mathcal{R}_{35}}{\mu_{3} \mathcal{R}_{25} \mathcal{R}_{55}} & v_{43} \frac{\mu_{3} \mathcal{R}_{15} \mathcal{R}_{45}}{\mu_{1} \mu_{4} \mu_{5} \mathcal{R}_{35}}
\end{array}\right) .
$$

In order to recover the double Myers-Perry black hole solution presented in [39], we reduce the set of solutions obtained in this way by restricting $\boldsymbol{V}$ to

$$
\boldsymbol{V}=\left(\begin{array}{ccc}
1 & v_{12} \frac{\mathcal{R}_{12} \mathcal{R}_{15}}{\rho^{2} \mathcal{R}_{13}} & 0 \\
1 & v_{22} \frac{\mathcal{R}_{24} \mathcal{R}_{45}}{\rho^{2} \mathcal{R}_{34}} & 0 \\
1 & 0 & 0 \\
1 & 0 & 0
\end{array}\right) .
$$

We immediately notice that this means in particular "trivializing" the solitons at $z=a_{2}$ and $z=a_{5}$. Hence, the solution obtained with this special choice of $\boldsymbol{V}$ can already be obtained from $n=2$ data. We should expect, however, a 4 -soliton transformation to be necessary in order to generate a (sufficiently general) double black hole solution, which suggests to explore the above more general solution. This will not be done here, and we return to the special case with the above restricted $\boldsymbol{V}$. Again, we obtain $\boldsymbol{\Gamma}$ from (3.18). Let $\hat{g}$ be the resulting solution (3.17), multiplied by $w$ to achieve the determinant condition. From Example 4.1, we obtain

$$
f_{(w)}=\rho^{-1}\left(\prod_{\substack{i=1 \\ i \neq 3}}^{5} \sqrt{\mathcal{R}_{i i}}\right)^{-1} \frac{\mathcal{R}_{12} \mathcal{R}_{15} \mathcal{R}_{24} \mathcal{R}_{45}}{\mathcal{R}_{14} \mathcal{R}_{25}},
$$

and then $\hat{f}$ via Corollary 4.1 and Proposition 4.2. Setting

$$
\begin{aligned}
& v_{12}=\frac{b\left(a_{1}-a_{3}\right)}{2\left(a_{1}-a_{2}\right)\left(a_{1}-a_{5}\right)}, \quad v_{22}=\frac{c\left(a_{3}-a_{4}\right)}{2\left(a_{2}-a_{4}\right)\left(a_{4}-a_{5}\right)}, \\
& \kappa=\left(4 \frac{\left(a_{1}-a_{2}\right)\left(a_{1}-a_{5}\right)\left(a_{2}-a_{4}\right)\left(a_{4}-a_{5}\right)}{\left(a_{1}-a_{4}\right)\left(a_{2}-a_{5}\right)}\right)^{2},
\end{aligned}
$$

with constants $b, c$, this results in the metric

$$
d s^{2}=-\frac{H_{2}}{H_{1}}\left(d t+\frac{\omega}{H_{2}} d \varphi\right)^{2}+\frac{\rho^{2} \mu_{3} H_{1}}{\mu_{2} \mu_{5} H_{2}} d \varphi^{2}+\frac{\mu_{2} \mu_{5}}{\mu_{3}} d \psi^{2}+k \frac{H_{1}}{F}\left(d \rho^{2}+d z^{2}\right),
$$


where

$$
\begin{aligned}
& H_{1}=M_{0}+b^{2} M_{1}+c^{2} M_{2}+b c M_{3}+b^{2} c^{2} M_{4}, \\
& H_{2}=\frac{\rho^{2}}{\mu_{2} \mu_{5}}\left(\frac{\mu_{1} \mu_{4}}{\rho^{2}} M_{0}-b^{2} \frac{\mu_{4}}{\mu_{1}} M_{1}-c^{2} \frac{\mu_{1}}{\mu_{4}} M_{2}-b c M_{3}+b^{2} c^{2} \frac{\rho^{2}}{\mu_{1} \mu_{4}} M_{4}\right), \\
& F=\mu_{3}^{2}\left(\mu_{1}-\mu_{4}\right)^{2}\left(\prod_{i=1}^{5} \mathcal{R}_{i i}\right) \frac{\mathcal{R}_{12} \mathcal{R}_{14}^{2} \mathcal{R}_{15} \mathcal{R}_{24} \mathcal{R}_{25}^{2} \mathcal{R}_{45}}{\mathcal{R}_{13} \mathcal{R}_{23} \mathcal{R}_{34} \mathcal{R}_{35}}, \\
& \omega=-2\left(\frac{\mu_{3}}{\mu_{2} \mu_{5}}\right)^{1 / 2}\left(b \mathfrak{R}_{1} \sqrt{M_{0} M_{1}}+c \Re_{4} \sqrt{M_{0} M_{2}}-b^{2} c \mathfrak{R}_{4} \sqrt{M_{1} M_{4}}-b c^{2} \mathfrak{R}_{1} \sqrt{M_{2} M_{4}}\right),
\end{aligned}
$$

with

$$
\begin{aligned}
& M_{0}=\mu_{2} \mu_{3}^{2} \mu_{5}\left(\mu_{1}-\mu_{4}\right)^{2} \mathcal{R}_{12}^{2} \mathcal{R}_{15}^{2} \mathcal{R}_{24}^{2} \mathcal{R}_{45}^{2}, \\
& M_{1}=\mu_{1}^{2} \mu_{2}^{2} \mu_{3} \mu_{5}^{2}\left(\mu_{1}-\mu_{3}\right)^{2} \mathcal{R}_{14}^{2} \mathcal{R}_{24}^{2} \mathcal{R}_{45}^{2} \\
& M_{2}=\mu_{2}^{2} \mu_{3} \mu_{4}^{2} \mu_{5}^{2}\left(\mu_{3}-\mu_{4}\right)^{2} \mathcal{R}_{12}^{2} \mathcal{R}_{14}^{2} \mathcal{R}_{15}^{2} \\
& M_{3}=2 \mu_{1} \mu_{2}^{2} \mu_{3} \mu_{4} \mu_{5}^{2}\left(\mu_{1}-\mu_{3}\right)\left(\mu_{3}-\mu_{4}\right) \mathcal{R}_{11} \mathcal{R}_{12} \mathcal{R}_{15} \mathcal{R}_{24} \mathcal{R}_{44} \mathcal{R}_{45}, \\
& M_{4}=\rho^{4} \mu_{1}^{2} \mu_{2}^{3} \mu_{4}^{2} \mu_{5}^{3}\left(\mu_{1}-\mu_{3}\right)^{2}\left(\mu_{1}-\mu_{4}\right)^{2}\left(\mu_{3}-\mu_{4}\right)^{2} .
\end{aligned}
$$

With obvious changes in notation, this is the metric obtained in [39].

Remark 4.4. It is plausible that one can start with the diagonal solution of the non-autonomous chiral model corresponding to a static triple black hole space-time (see (4.1) in [70]) and construct a space-time with three Myers-Perry black holes. This procedure should continue to produce solutions with an arbitrary number of rotating black holes.

\subsubsection{Bicycling black rings}

Let us start with the solution

$$
\tilde{g}=\operatorname{diag}\left(-\frac{\mu_{1} \mu_{5}}{\mu_{3} \mu_{7}}, \frac{\rho^{2} \mu_{3} \mu_{7}}{\mu_{2} \mu_{4} \mu_{6}}, \frac{\mu_{2} \mu_{4} \mu_{6}}{\mu_{1} \mu_{5}}\right)
$$

of (1.1), which corresponds to a static metric. Removal of a soliton at $z=a_{7}$ and an anti-soliton at $z=a_{1}$ from $\tilde{g}_{11}$, and a rescaling, leads to the seed metric

$$
g_{0}=w^{-1} \tilde{g} \operatorname{diag}\left(\frac{\mu_{7}^{2}}{\mu_{1}^{2}}, 1,1\right)=\operatorname{diag}\left(\frac{\mu_{5}}{\mu_{3}},-\frac{\rho^{2} \mu_{1} \mu_{3}}{\mu_{2} \mu_{4} \mu_{6}},-\frac{\mu_{2} \mu_{4} \mu_{6}}{\mu_{5} \mu_{7}}\right), \quad w=-\frac{\mu_{7}}{\mu_{1}},
$$

with the following solution of (4.1) (up to a constant factor),

$$
f_{0}=\frac{\mu_{2} \mu_{4} \mu_{6}}{\mu_{5} \mu_{7}} \frac{\mathcal{R}_{12} \mathcal{R}_{14} \mathcal{R}_{16} \mathcal{R}_{23} \mathcal{R}_{25} \mathcal{R}_{27} \mathcal{R}_{34} \mathcal{R}_{35} \mathcal{R}_{36} \mathcal{R}_{45} \mathcal{R}_{47} \mathcal{R}_{56} \mathcal{R}_{67}}{\sqrt{\mathcal{R}_{11}} \mathcal{R}_{13} \mathcal{R}_{22} \mathcal{R}_{24}^{2} \mathcal{R}_{26}^{2} \mathcal{R}_{33} \mathcal{R}_{44} \mathcal{R}_{46}^{2} \mathcal{R}_{55} \mathcal{R}_{57} \mathcal{R}_{66} \sqrt{\mathcal{R}_{77}}}
$$

We have $n=2$ and shall set

$$
\tilde{\boldsymbol{P}}=\operatorname{diag}\left(-\rho^{-1} \mu_{1}, \rho \mu_{7}^{-1}\right) .
$$

The solution of (3.8) is then given by (see Example 3.3)

$$
\boldsymbol{V}=\left(\begin{array}{ccc}
v_{11} \frac{\mathcal{R}_{13}}{\mathcal{R}_{15}} & v_{12} \frac{\mathcal{R}_{12} \mathcal{R}_{14} \mathcal{R}_{16}}{\mu_{1} \mathcal{R}_{11} \mathcal{R}_{13}} & v_{13} \frac{\mu_{1} \mathcal{R}_{15} \mathcal{R}_{17}}{\mathcal{R}_{12} \mathcal{R}_{14} \mathcal{R}_{16}} \\
v_{21} \frac{\mu_{3} \mathcal{R}_{57}}{\mu_{5} \mathcal{R}_{37}} & v_{22} \frac{\mu_{2} \mu_{4} \mu_{6} \mathcal{R}_{17} \mathcal{R}_{37}}{\mu_{1} \mu_{3} \mathcal{R}_{27} \mathcal{R}_{47} \mathcal{R}_{67}} & v_{23} \frac{\mu_{5} \mathcal{R}_{27} \mathcal{R}_{47} \mathcal{R}_{67}}{\mu_{2} \mu_{4} \mu_{6} \mathcal{R}_{57} \mathcal{R}_{77}}
\end{array}\right)
$$


Without restriction of generality, we can set $v_{11}=v_{21}=1$. But we do restrict the class of solutions by setting

$$
v_{12}=v_{23}=0 \text {. }
$$

Again, the solution $\boldsymbol{\Gamma}$ of the Stein equation is obtained from (3.18). The resulting solution (3.17) of the non-autonomous chiral model has to be modified to $\hat{g}=w g$, with $w$ as given above, in order to achieve the determinant condition. We have (disregarding a constant factor)

$$
f_{(w)}=\rho^{-1} \frac{\mathcal{R}_{17}}{\sqrt{\mathcal{R}_{11} \mathcal{R}_{77}}}
$$

and, from Corollary 4.1 and Proposition 4.2,

$$
\hat{f}=\kappa \rho^{-5} w f_{0} f_{(w)}^{-3} \frac{(\operatorname{det} \tilde{\boldsymbol{P}})^{2} \operatorname{det} \boldsymbol{\Gamma}}{\operatorname{det}\left(\boldsymbol{I}+\tilde{\boldsymbol{P}}^{2}\right)},
$$

which results in a lengthy expression. Setting

$$
v_{13}=c_{1}, \quad v_{22}=b_{2} \frac{\left(a_{7}-a_{4}\right)\left(a_{7}-a_{5}\right)\left(a_{7}-a_{6}\right)}{\left(a_{7}-a_{1}\right)\left(a_{7}-a_{3}\right)^{2}}, \quad \kappa=4\left(a_{7}-a_{1}\right)^{2},
$$

we obtain the metric ${ }^{19}$

$$
\begin{aligned}
d s^{2}= & -\frac{H_{2}}{H_{1}}\left(d t-\frac{\omega_{1}}{H_{2}} d \varphi-\frac{\omega_{2}}{H_{2}} d \psi\right)^{2} \\
& +\frac{1}{H_{2}}\left(G_{1} d \varphi^{2}+G_{2} d \psi^{2}-2 J d \varphi d \psi\right)+\mathcal{P} H_{1}\left(d \rho^{2}+d z^{2}\right)
\end{aligned}
$$

where

$$
\begin{aligned}
H_{1}= & M_{0}+c_{1}^{2} M_{1}+b_{2}^{2} M_{2}-b_{2}^{2} c_{1}^{2} M_{3}, \\
H_{2}= & \frac{\mu_{5}}{\mu_{3}}\left(\frac{\mu_{1}}{\mu_{7}} M_{0}-c_{1}^{2} \frac{\rho^{2}}{\mu_{1} \mu_{7}} M_{1}-b_{2}^{2} \frac{\mu_{1} \mu_{7}}{\rho^{2}} M_{2}-b_{2}^{2} c_{1}^{2} \frac{\mu_{7}}{\mu_{1}} M_{3}\right), \\
G_{1}= & \frac{\rho^{2} \mu_{1} \mu_{5}}{\mu_{2} \mu_{4} \mu_{6}}\left(M_{0}-c_{1}^{2} \frac{\rho^{2}}{\mu_{1}^{2}} M_{1}+b_{2}^{2} M_{2}+b_{2}^{2} c_{1}^{2} \frac{\rho^{2}}{\mu_{1}^{2}} M_{3}\right), \\
G_{2}= & \frac{\mu_{2} \mu_{4} \mu_{6}}{\mu_{3} \mu_{7}}\left(M_{0}+c_{1}^{2} M_{1}-b_{2}^{2} \frac{\mu_{7}^{2}}{\rho^{2}} M_{2}+b_{2}^{2} c_{1}^{2} \frac{\mu_{7}^{2}}{\rho^{2}} M_{3}\right), \\
\omega_{1}= & b_{2} \frac{\mathcal{R}_{77}}{\mu_{7}}\left(\frac{\mu_{1} \mu_{5}}{\mu_{2} \mu_{4} \mu_{6}}\right)^{1 / 2}\left(\sqrt{M_{0} M_{2}}-c_{1}^{2} \frac{\rho}{\mu_{1}} \sqrt{M_{1} M_{3}}\right), \\
\omega_{2}= & c_{1} \frac{\mathcal{R}_{11}}{\mu_{1}}\left(\frac{\mu_{2} \mu_{4} \mu_{6}}{\rho^{2} \mu_{3} \mu_{7}}\right)^{1 / 2}\left(\sqrt{M_{0} M_{1}}-b_{2}^{2} \frac{\mu_{7}}{\rho} \sqrt{M_{2} M_{3}}\right), \\
J= & b_{2} c_{1} \rho^{2} \mu_{1} \mu_{2} \mu_{3} \mu_{4} \mu_{5}^{2} \mu_{6}\left(\mu_{3}-\mu_{7}\right)^{2}\left(\mu_{4}-\mu_{7}\right)\left(\mu_{5}-\mu_{7}\right)\left(\mu_{6}-\mu_{7}\right) \\
& \times \mathcal{R}_{11} \mathcal{R}_{12} \mathcal{R}_{13} \mathcal{R}_{14} \mathcal{R}_{15}^{2} \mathcal{R}_{16} \mathcal{R}_{17} \mathcal{R}_{27} \mathcal{R}_{77},
\end{aligned}
$$

and

$$
\begin{aligned}
& M_{0}=\mu_{4} \mu_{5}^{3} \mu_{6} \mu_{7}\left(\mu_{3}-\mu_{7}\right)^{4} \mathcal{R}_{12}^{2} \mathcal{R}_{13}^{2} \mathcal{R}_{14}^{2} \mathcal{R}_{16}^{2} \mathcal{R}_{17}^{2} \mathcal{R}_{27}^{2} \\
& M_{1}=\rho^{2} \mu_{1}^{2} \mu_{2} \mu_{3} \mu_{4}^{2} \mu_{5} \mu_{6}^{2}\left(\mu_{1}-\mu_{7}\right)^{2}\left(\mu_{3}-\mu_{7}\right)^{4} \mathcal{R}_{15}^{4} \mathcal{R}_{17}^{2} \mathcal{R}_{27}^{2},
\end{aligned}
$$

\footnotetext{
${ }^{19}$ Here we used $a_{i}-a_{j}=\left(\mu_{i}-\mu_{j}\right)\left(\rho^{2}+\mu_{i} \mu_{j}\right) /\left(2 \mu_{i} \mu_{j}\right)$ to eliminate $a_{i}-a_{j}$.
} 


$$
\begin{aligned}
& M_{2}=\rho^{4} \mu_{1} \mu_{2} \mu_{3}^{2} \mu_{5}^{2} \mu_{7}\left(\mu_{4}-\mu_{7}\right)^{2}\left(\mu_{5}-\mu_{7}\right)^{2}\left(\mu_{6}-\mu_{7}\right)^{2} \mathcal{R}_{12}^{2} \mathcal{R}_{13}^{2} \mathcal{R}_{14}^{2} \mathcal{R}_{16}^{2} \\
& M_{3}=\rho^{4} \mu_{1}^{3} \mu_{2}^{2} \mu_{3}^{3} \mu_{4} \mu_{6}\left(\mu_{4}-\mu_{7}\right)^{2}\left(\mu_{5}-\mu_{7}\right)^{2}\left(\mu_{6}-\mu_{7}\right)^{2} \mathcal{R}_{15}^{4} \mathcal{R}_{17}^{2} \\
& \mathcal{P}=\frac{\mu_{2}}{\mu_{1} \mu_{5}^{4} \mu_{7}\left(\mu_{3}-\mu_{7}\right)^{4}} \frac{\mathcal{R}_{23} \mathcal{R}_{25} \mathcal{R}_{34} \mathcal{R}_{35} \mathcal{R}_{36} \mathcal{R}_{45} \mathcal{R}_{47} \mathcal{R}_{56} \mathcal{R}_{57} \mathcal{R}_{13} \mathcal{R}_{14} \mathcal{R}_{15}^{2} \mathcal{R}_{16} \mathcal{R}_{17} \mathcal{R}_{24}^{2} \mathcal{R}_{26}^{2} \mathcal{R}_{27} \mathcal{R}_{37}^{2} \mathcal{R}_{46}^{2}}{}
\end{aligned}
$$

With obvious changes in notation, this is the "bicycling" black bi-ring solution obtained in [23] (also see [47]), except for the fact that we have a minus sign instead of a plus in the expressions for $\omega_{1}$ and $\omega_{2}$.

\section{$5 \quad$ Final remarks}

We presented a general formulation of binary Darboux-type transformations in the bidifferential calculus framework. Whenever a PDDE can be cast into the form (2.2) or (2.3), Theorem 2.1 can be applied and it will typically generate a large class of exact solutions. Meanwhile a bidifferential calculus formulation is available for quite a number of integrable PDDEs.

We elaborated this general result for the case of the non-autonomous chiral model, considerably extending previous results in [15]. We also presented conditions that, imposed on the matrix data that determine the general class of solutions, guarantee that the resulting solution of the non-autonomous chiral model is symmetric (or Hermitian). If the solution is also real, then it is known to determine a Ricci-flat metric, i.e., a solution of the vacuum Einstein equations, dimensionally reduced to two dimensions. We essentially solved the equations resulting from the assumptions in Theorem 2.1 in the case of a diagonal seed metric, though not yet the $\boldsymbol{V}$-equations in sufficient generality if $\tilde{\boldsymbol{P}}$ is non-diagonal (but see Examples 4.3 and 4.4). All this provides a working recipe to compute quite easily solutions of the vacuum Einstein equations. In particular, in the four-dimensional case we recovered (multi-) Kerr-NUT (in a different way than in [15]) and the $\delta=2$ Tomimatsu-Sato solution. In the five-dimensional case we recovered single and double Myers-Perry black holes, the "black saturn" and the "bicycling black ring" solutions. The more general solutions still have to be explored. In view of the complexity of the latter solutions, it is certainly an advantage to have now an independent method at our disposal to derive, verify or generalize them. Surely further important solutions of Einstein's equations in $D \geq 4$ space-time dimensions can be recovered using this method and there is a chance to discover interesting new solutions. We concentrated on examples in the stationary case $\epsilon=1$, but developed the formalism as well for the wave case $\epsilon=-1[2,4]$. It is not difficult to recover relevant examples in this case too.

The recipe to construct solutions of the non-autonomous chiral model and the dimensionally reduced vacuum Einstein equations, obtained from Theorem 2.1, is - not surprisingly - a variant, a sort of matrix version, of the well-known method of Belinski and Zakharov [2-4].

One should look for suitable ways to spot physically relevant solutions within the plethora of solutions. How are desired properties of solutions, like asymptotic flatness, absence of naked singularities and proper axis conditions encoded in the (matrix) data that determine a solution? Here the rod structure analysis $[2,9,27,36]$, developed for the Belinski-Zakharov approach and frequently used, is of great help.

Section 3 also paved the way toward a treatment of other reductions of the non-autonomous chiral model, which, e.g., are relevant in the Einstein-Maxwell case and supergravity theories.

In this work we only elaborated Theorem 2.1 for a particular example of an integrable equation in the bidifferential calculus framework. Although we already applied a more restricted version of it previously to several other integrable equations, it will be worth to reconsider them and to also explore further equations, using the much more general solution-generating tool we now have at our disposal. Furthermore, it should be clarified whether, e.g., the examples in [63-65] fit into the framework of Theorem 2.1. We should also mention that Sylvester equations, like 
those that arise from (2.7), and more generally operator versions of them, are ubiquitous in the theory of integrable systems. In particular, they are related to a Riemann-Hilbert factorization problem [66] and they are at the roots of Marchenko's operator approach [53].

\section{Appendix A. Addendum to Example 4.1}

From (4.3) we find that

$$
\mathfrak{f}[\rho] \propto \rho^{1 / 4}, \quad \mathfrak{f}\left[w^{k}\right] \propto \mathfrak{f}[w]^{k^{2}}, \quad \mathfrak{f}\left[w_{1} w_{2}\right] \propto \mathfrak{f}\left[w_{1}\right] \mathfrak{f}\left[w_{2}\right] \mathfrak{F}\left[w_{1}, w_{2}\right],
$$

where $\mathfrak{F}\left[w_{1}, w_{2}\right]$ has to solve

$$
\begin{aligned}
\left(\ln \mathfrak{F}\left[w_{1}, w_{2}\right]\right)_{\rho} & =\frac{\rho}{2}\left(\left(\ln w_{1}\right)_{\rho}\left(\ln w_{2}\right)_{\rho}-\epsilon\left(\ln w_{1}\right)_{z}\left(\ln w_{2}\right)_{z}\right), \\
\left(\ln \mathfrak{F}\left[w_{1}, w_{2}\right]\right)_{z} & =\frac{\rho}{2}\left(\left(\ln w_{1}\right)_{\rho}\left(\ln w_{2}\right)_{z}+\left(\ln w_{1}\right)_{z}\left(\ln w_{2}\right)_{\rho}\right)
\end{aligned}
$$

(also see [47]). It is easy to verify that

$$
\mathfrak{F}\left[w_{1} \cdots w_{r}, w_{1}^{\prime} \cdots w_{s}^{\prime}\right] \propto \prod_{i=1}^{r} \prod_{j=1}^{s} \mathfrak{F}\left[w_{i}, w_{j}^{\prime}\right]
$$

In particular, $\mathfrak{F}\left[w_{1}^{k}, w_{2}^{l}\right] \propto \mathfrak{F}\left[w_{1}, w_{2}\right]^{k l}$. Furthermore, we have

$$
\mathfrak{F}\left[\rho^{k} w_{1}, \rho^{l} w_{2}\right] \propto \rho^{k l / 2} w_{1}^{l / 2} w_{2}^{k / 2} \mathfrak{F}\left[w_{1}, w_{2}\right] .
$$

It follows that

$$
\mathfrak{f}\left[\rho^{k} w\right] \propto \rho^{k^{2} / 4} w^{k / 2} \mathfrak{f}[w], \quad \mathfrak{f}\left[\tilde{\mu}_{1} \cdots \tilde{\mu}_{r}\right] \propto\left(\prod_{k=1}^{r} \mathfrak{f}\left[\tilde{\mu}_{k}\right]\right)\left(\prod_{i<j} \mathfrak{F}\left[\tilde{\mu}_{i}, \tilde{\mu}_{j}\right]\right),
$$

and

$$
\mathfrak{f}\left[\frac{\tilde{\mu}_{1} \cdots \tilde{\mu}_{r}}{\tilde{\mu}_{1}^{\prime} \cdots \tilde{\mu}_{s}^{\prime}}\right] \propto \frac{\mathfrak{f}\left[\tilde{\mu}_{1} \cdots \tilde{\mu}_{r}\right] \mathfrak{f}\left[\tilde{\mu}_{1}^{\prime} \cdots \tilde{\mu}_{r}^{\prime}\right]}{\prod_{i}^{r} \prod_{j}^{s} \mathfrak{F}\left[\tilde{\mu}_{i}, \tilde{\mu}_{j}^{\prime}\right]}
$$

from which the main result in Example 4.1 is easily deduced.

\section{Appendix B. Some proofs}

Proof of Proposition 4.1. Using $(\ln \operatorname{det} Y)_{\rho}=\operatorname{tr}\left(Y_{\rho} Y^{-1}\right)$ for an invertible (and differentiable) matrix function $Y$, we obtain

$$
\begin{aligned}
(\ln f)_{\rho}= & \left(\ln f_{0}\right)_{\rho}+\operatorname{tr}\left(-\rho^{-1} \boldsymbol{I}+\tilde{\boldsymbol{X}}_{\rho} \tilde{\boldsymbol{X}}^{-1}+\tilde{\boldsymbol{P}}_{\rho} \tilde{\boldsymbol{P}}^{-1}+\tilde{\boldsymbol{Q}}_{\rho} \tilde{\boldsymbol{Q}}^{-1}-\epsilon \tilde{\boldsymbol{P}}_{\rho} \tilde{\boldsymbol{P}}\left(1+\epsilon \tilde{\boldsymbol{P}}^{2}\right)^{-1}\right. \\
& \left.-\epsilon \tilde{\boldsymbol{Q}}_{\rho} \tilde{\boldsymbol{Q}}\left(1+\epsilon \tilde{\boldsymbol{Q}}^{2}\right)^{-1}\right), \\
(\ln f)_{z}= & \left(\ln f_{0}\right)_{z}+\operatorname{tr}\left(\tilde{\boldsymbol{X}}_{z} \tilde{\boldsymbol{X}}^{-1}+\tilde{\boldsymbol{P}}_{z} \tilde{\boldsymbol{P}}^{-1}+\tilde{\boldsymbol{Q}}_{z} \tilde{\boldsymbol{Q}}^{-1}-\epsilon \tilde{\boldsymbol{P}}_{z} \tilde{\boldsymbol{P}}\left(1+\epsilon \tilde{\boldsymbol{P}}^{2}\right)^{-1}\right. \\
& \left.-\epsilon \tilde{\boldsymbol{Q}}_{z} \tilde{\boldsymbol{Q}}\left(1+\epsilon \tilde{\boldsymbol{Q}}^{2}\right)^{-1}\right),
\end{aligned}
$$


for the expression of $f$ in Proposition 4.1. In order to verify that (4.1) holds, we have to show that these expressions equal the corresponding right hand sides of (4.1), evaluated with

$$
\begin{aligned}
\mathcal{U}= & -\rho \tilde{\phi}_{z}=-\rho\left[\tilde{\phi}_{0, z}+\left(\boldsymbol{U} \tilde{\boldsymbol{X}}^{-1} \boldsymbol{V}\right)_{z}\right] \\
& =\mathcal{U}_{0}-\rho\left(\boldsymbol{U}_{z} \tilde{\boldsymbol{X}}^{-1} \boldsymbol{V}-\boldsymbol{U} \tilde{\boldsymbol{X}}^{-1} \tilde{\boldsymbol{X}}_{z} \tilde{\boldsymbol{X}}^{-1} \boldsymbol{V}+\boldsymbol{U} \tilde{\boldsymbol{X}}^{-1} \boldsymbol{V}_{z}\right), \\
\mathcal{V}= & \mathcal{V}_{0}+\epsilon\left[\rho\left(\boldsymbol{U}_{\rho} \tilde{\boldsymbol{X}}^{-1} \boldsymbol{V}-\boldsymbol{U} \tilde{\boldsymbol{X}}^{-1} \tilde{\boldsymbol{X}}_{\rho} \tilde{\boldsymbol{X}}^{-1} \boldsymbol{V}+\boldsymbol{U} \tilde{\boldsymbol{X}}^{-1} \boldsymbol{V}_{\rho}\right)+\boldsymbol{U} \tilde{\boldsymbol{X}}^{-1} \boldsymbol{V}\right],
\end{aligned}
$$

where $\mathcal{U}_{0}=-\rho \tilde{\phi}_{0, z}$ and $\mathcal{V}_{0}=\epsilon\left(\rho \tilde{\phi}_{0, \rho}+\tilde{\phi}_{0}\right) .{ }^{20}$ We work on the right hand sides of (4.1) and, as intermediate steps, we consider $\operatorname{tr}\left(\mathcal{U}^{2}-\mathcal{U}_{0}^{2}\right), \operatorname{tr}\left(\mathcal{V}^{2}-\mathcal{V}_{0}^{2}\right)$ and $\operatorname{tr}\left(\mathcal{U} \mathcal{V}-\mathcal{U}_{0} \mathcal{V}_{0}\right)$ separately. First we eliminate derivatives of $\phi_{0}$ with the help of (3.7) and (3.8). Then we eliminate $\boldsymbol{V} \boldsymbol{U}$ using the Sylvester equation, and $\boldsymbol{V}_{z} \boldsymbol{U}, \boldsymbol{V}_{\rho} \boldsymbol{U}$ via (3.14). Rewriting (3.14) with the help of (3.10), we obtain a version that allows us to also replace all occurencies of $\boldsymbol{V} \boldsymbol{U}_{z}$ and $\boldsymbol{V} \boldsymbol{U}_{\rho}$. Several times one has to exploit the cyclicity of the trace. Finally we use

$$
\tilde{\boldsymbol{P}}_{\rho}=\rho^{-1} \tilde{\boldsymbol{P}}\left(\boldsymbol{I}-\epsilon \tilde{\boldsymbol{P}}^{2}\right)\left(\boldsymbol{I}+\epsilon \tilde{\boldsymbol{P}}^{2}\right)^{-1}, \quad \tilde{\boldsymbol{P}}_{z}=2 \epsilon \rho^{-1} \tilde{\boldsymbol{P}}^{2}\left(\boldsymbol{I}+\epsilon \tilde{\boldsymbol{P}}^{2}\right)^{-1}
$$

(which follows from (3.3)), and the corresponding equations for $\tilde{\boldsymbol{Q}}$, to show that (4.1) holds.

Proof of Proposition 4.2. Using $\hat{\mathcal{U}}=\mathcal{U}+\rho(\ln w)_{\rho} I$ and $\hat{\mathcal{V}}=\mathcal{V}+\rho(\ln w)_{z} I$, we have

$$
\operatorname{tr}\left(\hat{\mathcal{U}}^{2}-\mathcal{U}^{2}\right)=2 \rho(\ln w)_{\rho} \operatorname{tr} \mathcal{U}+m \rho^{2}(\ln w)_{\rho}^{2}=2 \rho^{2}(\ln w)_{\rho}(\ln \operatorname{det} g)_{\rho}+m \rho^{2}(\ln w)_{\rho}^{2},
$$

and a corresponding expression for $\operatorname{tr}\left(\hat{\mathcal{V}}^{2}-\mathcal{V}^{2}\right)$. Furthermore,

$$
\operatorname{tr}(\hat{\mathcal{U}} \hat{\mathcal{V}}-\mathcal{U V})=\rho^{2}\left((\ln w)_{\rho}(\ln \operatorname{det} g)_{z}+(\ln w)_{z}(\ln \operatorname{det} g)_{\rho}+m(\ln w)_{\rho}(\ln w)_{z}\right) .
$$

With the help of (4.1) we obtain

$$
\begin{aligned}
\left(\ln \frac{\hat{f}}{f}\right)_{\rho} & =\frac{1}{4 \rho} \operatorname{tr}\left(\hat{\mathcal{U}}^{2}-\mathcal{U}^{2}-\epsilon\left(\hat{\mathcal{V}}^{2}-\mathcal{V}^{2}\right)\right) \\
& =\frac{\rho}{2}\left((\ln w)_{\rho}(\ln \operatorname{det} g)_{\rho}-\epsilon(\ln w)_{z}(\ln \operatorname{det} g)_{z}\right)+m \frac{\rho}{4}\left((\ln w)_{\rho}^{2}-\epsilon(\ln w)_{z}^{2}\right) \\
\left(\ln \frac{\hat{f}}{f}\right)_{z} & =\frac{1}{2 \rho} \operatorname{tr}(\hat{\mathcal{U}} \hat{\mathcal{V}}-\mathcal{U} \mathcal{V}) \\
& =\frac{\rho}{2}\left((\ln w)_{\rho}(\ln \operatorname{det} g)_{z}+(\ln w)_{z}(\ln \operatorname{det} g)_{\rho}\right)+m \frac{\rho}{2}(\ln w)_{\rho}(\ln w)_{z} .
\end{aligned}
$$

Next we use

$$
\left(\ln \left(\rho f_{(w)}\right)\right)_{\rho}=\frac{\rho}{4}\left((\ln w)_{\rho}^{2}-\epsilon(\ln w)_{z}^{2}\right), \quad\left(\ln \left(\rho f_{(w)}\right)\right)_{z}=\frac{\rho}{2}(\ln w)_{\rho}(\ln w)_{z},
$$

and the corresponding equations for $f_{(\operatorname{det} g)}$ and $f_{(w \operatorname{det} g)}$, to deduce that

$$
\begin{aligned}
& \left(\ln \frac{f_{(w \operatorname{det} g)}}{\rho f_{(w)} f_{(\operatorname{det} g)}}\right)_{\rho}=\frac{\rho}{2}\left((\ln w)_{\rho}(\ln \operatorname{det} g)_{\rho}-\epsilon(\ln w)_{z}(\ln \operatorname{det} g)_{z}\right), \\
& \left(\ln \frac{f_{(w \operatorname{det} g)}}{\rho f_{(w)} f_{(\operatorname{det} g)}}\right)_{z}=\frac{\rho}{2}\left((\ln w)_{\rho}(\ln \operatorname{det} g)_{z}+(\ln w)_{z}(\ln \operatorname{det} g)_{\rho}\right) .
\end{aligned}
$$

Inserting the last expressions in our previous results, we obtain (4.4) by integration. Let us now assume that $\operatorname{det} \hat{g}=-\epsilon \rho^{2}$, and thus $\operatorname{det} g=-\epsilon \rho^{2} w^{-m}$. With

$$
f_{\left(\rho^{2} w^{k}\right)} \propto w^{k}\left(\rho f_{(w)}\right)^{k^{2}}
$$

(see Example 4.1), (4.5) results from (4.4).

\footnotetext{
${ }^{20}$ This is quite a tour de force and a more elegant proof would be desirable.
} 


\section{References}

[1] Arsie A., Lorenzoni P., F-manifolds with eventual identities, bidifferential calculus and twisted LenardMagri chains, Int. Math. Res. Not., to appear, arXiv:1110.2461.

[2] Belinski V.A., Verdaguer E., Gravitational solitons, Cambridge Monographs on Mathematical Physics, Cambridge University Press, Cambridge, 2001.

[3] Belinskiŭ V.A., Sakharov V.E., Stationary gravitational solitons with axial symmetry, Sov. Phys. JETP 50 (1979), 1-9.

[4] Belinskiǔ V.A., Zakharov V.E., Integration of the Einstein equations by means of the inverse scattering problem technique and construction of exact soliton solutions, Sov. Phys. JETP 48 (1978), 985-994.

[5] Breitenlohner P., Maison D., Gibbons G., 4-dimensional black holes from Kaluza-Klein theories, Comm. Math. Phys. 120 (1988), 295-333.

[6] Camacaro J., Cariñena J., Alternative Lie algebroid structures and bi-differential calculi, in Applied Differential Geometry and Mechanics, Editors W. Sarlet, F. Cantrijn, University of Gent, 2003, 1-20.

[7] Chavchanidze G., Non-Noether symmetries in Hamiltonian dynamical systems, Mem. Differential Equations Math. Phys. 36 (2005), 81-134, math-ph/0405003.

[8] Chen Y., Hong K., Teo E., Unbalanced Pomeransky-Sen'kov black ring, Phys. Rev. D 84 (2011), 084030, 11 pages, arXiv:1108.1849.

[9] Chen Y., Teo E., Rod-structure classification of gravitational instantons with $U(1) \times U(1)$ isometry, Nuclear Phys. B 838 (2010), 207-237, arXiv:1004.2750.

[10] Chruściel P., Cortier J., Maximal analytic extensions of the Emparan-Reall black ring, J. Phys. Conf. Ser. 229 (2010), 012030, 4 pages, arXiv:0807.2309.

[11] Chruściel P.T., Eckstein M., Szybka S.J., On smoothness of black saturns, J. High Energy Phys. 2010 (2010), no. 11, 048, 39 pages, arXiv:1007.3668.

[12] Cieśliński J.L., Algebraic construction of the Darboux matrix revisited, J. Phys. A: Math. Theor. 42 (2009), 404003, 40 pages, arXiv:0904.3987.

[13] Crampin M., Sarlet W., Thompson G., Bi-differential calculi, bi-Hamiltonian systems and conformal Killing tensors, J. Phys. A: Math. Gen. 33 (2000), 8755-8770.

[14] de Souza E., Bhattacharyya S.P., Controllability, observability and the solution of $A X-X B=C$, Linear Algebra Appl. 39 (1981), 167-188.

[15] Dimakis A., Kanning N., Müller-Hoissen F., The non-autonomous chiral model and the Ernst equation of general relativity in the bidifferential calculus framework, SIGMA 7 (2011), 118, 27 pages, arXiv:1106.4122.

[16] Dimakis A., Müller-Hoissen F., Bi-differential calculi and integrable models, J. Phys. A: Math. Gen. 33 (2000), 957-974, math-ph/9908015.

[17] Dimakis A., Müller-Hoissen F., Bicomplexes and integrable models, J. Phys. A: Math. Gen. 33 (2000), 6579-6591, nlin.SI/0006029.

[18] Dimakis A., Müller-Hoissen F., Bidifferential calculus approach to AKNS hierarchies and their solutions, SIGMA 6 (2010), 055, 27 pages, arXiv:1004.1627.

[19] Dimakis A., Müller-Hoissen F., Bidifferential graded algebras and integrable systems, Discrete Contin. Dyn. Syst. (2009), suppl., 208-219, arXiv:0805.4553.

[20] Dimakis A., Müller-Hoissen F., Solutions of matrix NLS systems and their discretizations: a unified treatment, Inverse Problems 26 (2010), 095007, 55 pages, arXiv:1001.0133.

[21] Economou A., Tsoubelis D., Multiple-soliton solutions of Einstein's equations, J. Math. Phys. 30 (1989), 1562-1569.

[22] Elvang H., Figueras P., Black saturn, J. High Energy Phys. 2007 (2007), no. 5, 050, 48 pages, hep-th/0701035.

[23] Elvang H., Rodriguez M.J., Bicycling black rings, J. High Energy Phys. 2008 (2008), no. 4, 045, 30 pages, arXiv:0712.2425.

[24] Emparan R., Reall H.S., A rotating black ring solution in five dimensions, Phys. Rev. Lett. 88 (2002), 101101, 4 pages, hep-th/0110260.

[25] Emparan R., Reall H.S., Black holes in higher dimensions, Living Rev. Relativ. 11 (2008), 6, 87 pages, arXiv:0801.3471. 
[26] Emparan R., Reall H.S., Black rings, Classical Quantum Gravity 23 (2006), R169-R197, hep-th/0608012.

[27] Emparan R., Reall H.S., Generalized Weyl solutions, Phys. Rev. D 65 (2002), 084025, 26 pages, hep-th/0110258.

[28] Evslin J., Krishnan C., The black di-ring: an inverse scattering construction, Classical Quantum Gravity 26 (2009), 125018, 13 pages, arXiv:0706.1231.

[29] Figueras P., A black ring with a rotating 2-sphere, J. High Energy Phys. 2005 (2005), no. 7, 039, 9 pages, hep-th/0505244.

[30] Figueras P., Jamsin E., Rocha J.V., Virmani A., Integrability of five-dimensional minimal supergravity and charged rotating black holes, Classical Quantum Gravity 27 (2010), 135011, 37 pages, arXiv:0912.3199.

[31] Frölicher A., Nijenhuis A., Theory of vector-valued differential forms. I. Derivations in the graded ring of differential forms, Proc. Koninkl. Ned. Acad. Wetensch. Ser. A 59 (1956), 338-359.

[32] Frolov V.P., Goswami R., Surface geometry of 5D black holes and black rings, Phys. Rev. D 75 (2007), 124001, 11 pages, gr-qc/0612033.

[33] Gauntlett J.P., Gutowski J.B., Concentric black rings, Phys. Rev. D 71 (2005), 025013, 7 pages, hep-th/0408010.

[34] Griffiths J.B., Colliding plane waves in general relativity, Oxford Mathematical Monographs, Oxford Science Publications, The Clarendon Press, Oxford University Press, New York, 1991.

[35] Gu C., Hu H., Zhou Z., Darboux transformations in integrable systems. Theory and their applications to geometry, Mathematical Physics Studies, Vol. 26, Springer, Dordrecht, 2005.

[36] Harmark T., Stationary and axisymmetric solutions of higher-dimensional general relativity, Phys. Rev. D 70 (2004), 124002, 25 pages, hep-th/0408141.

[37] Hartwig R.E., Resultants and the solution of $A X-X B=-C$, SIAM J. Appl. Math. 23 (1972), $104-117$.

[38] Hearon J.Z., Nonsingular solutions of $T A-B T=C$, Linear Algebra Appl. 16 (1977), 57-63.

[39] Herdeiro C., Rebelo C., Zilhão M., Costa M., A double Myers-Perry black hole in five dimensions, J. High Energy Phys. 2008 (2008), no. 7, 009, 24 pages, arXiv:0805.1206.

[40] Hollands S., Yazadjiev S., Uniqueness theorem for 5-dimensional black holes with two axial Killing fields, Comm. Math. Phys. 283 (2008), 749-768, arXiv:0707.2775.

[41] Hong K., Teo E., A new form of the C-metric, Classical Quantum Gravity 20 (2003), 3269-3277, gr-qc/0305089.

[42] Horowitz G. (Editor), Black holes in higher dimensions, Cambridge University Press, Cambridge, 2012.

[43] Hoskisson J., Explorations of four and five dimensional black hole spacetimes, Ph.D. thesis, Durham University, 2009, available at http://etheses.dur.ac.uk/2115/.

[44] Hu Q., Cheng D., The polynomial solution to the Sylvester matrix equation, Appl. Math. Lett. 19 (2006), 859-864.

[45] Iguchi H., Izumi K., Mishima T., Systematic solution-generation of five-dimensional black holes, Progr. Theoret. Phys. Suppl. 189 (2011), 93-125, arXiv:1106.0387.

[46] Iguchi H., Mishima T., Solitonic generation of vacuum solutions in five-dimensional general relativity, Phys. Rev. D $\mathbf{7 4}$ (2006), 024029, 17 pages, hep-th/0605090.

[47] Izumi K., Orthogonal black di-ring solution, Progr. Theoret. Phys. 119 (2008), 757-774, arXiv:0712.0902.

[48] Klein C., Richter O., Ernst equation and Riemann surfaces. Analytical and numerical methods, Lecture Notes in Physics, Vol. 685, Springer-Verlag, Berlin, 2005.

[49] Kodama H., Hikida W., Global structure of the Zipoy-Voorhees-Weyl spacetime and the $\delta=2$ TomimatsuSato spacetime, Classical Quantum Gravity 20 (2003), 5121-5140, gr-qc/0304064.

[50] Kramer D., Neugebauer G., The superposition of two Kerr solutions, Phys. Lett. A 75 (1980), $259-261$.

[51] Lorenzoni P., Flat bidifferential ideals and semi-Hamiltonian PDEs, J. Phys. A: Math. Gen. 39 (2006), 13701-13715, nlin.SI/0604053.

[52] Lorenzoni P., Magri F., A cohomological construction of integrable hierarchies of hydrodynamic type, Int. Math. Res. Not. 2005 (2005), no. 34, 2087-2100, nlin.SI/0504064.

[53] Marchenko V.A., Nonlinear equations and operator algebras, Mathematics and its Applications (Soviet Series), Vol. 17, D. Reidel Publishing Co., Dordrecht, 1988. 
[54] Matveev V.B., Salle M.A., Darboux transformations and solitons, Springer Series in Nonlinear Dynamics, Springer-Verlag, Berlin, 1991.

[55] Mishima T., Iguchi H., New axisymmetric stationary solutions of five-dimensional vacuum Einstein equations with asymptotic flatness, Phys. Rev. D 73 (2006), 044030, 6 pages, hep-th/0504018.

[56] Myers R.C., Myers-Perry black holes, in Black Holes in Higher Dimensions, Editor G. Horowitz, Cambridge University Press, Cambridge, 2012, 101-133, arXiv:1111.1903.

[57] Myers R.C., Perry M.J., Black holes in higher-dimensional space-times, Ann. Physics 172 (1986), 304-347.

[58] Nakamura Y., Symmetries of stationary axially symmetric vacuum Einstein equations and the new family of exact solutions, J. Math. Phys. 24 (1983), 606-609.

[59] Nimmo J.J.C., Gilson C., Ohta Y., Applications of Darboux transformations to the self-dual Yang-Mills equations, Theoret. and Math. Phys. 122 (2000), 239-246.

[60] Pomeransky A.A., Complete integrability of higher-dimensional Einstein equations with additional symmetry and rotating black holes, Phys. Rev. D 73 (2006), 044004, 5 pages, hep-th/0507250.

[61] Pomeransky A.A., Sen'kov R.A., Black ring with two angular momenta, hep-th/0612005.

[62] Rogers C., Schief W.K., Bäcklund and Darboux transformations. Geometry and modern applications in soliton theory, Cambridge Texts in Applied Mathematics, Cambridge University Press, Cambridge, 2002.

[63] Sakhnovich A.L., Dressing procedure for solutions of nonlinear equations and the method of operator identities, Inverse Problems 10 (1994), 699-710.

[64] Sakhnovich A.L., Generalized Bäcklund-Darboux transformation: spectral properties and nonlinear equations, J. Math. Anal. Appl. 262 (2001), 274-306.

[65] Sakhnovich A.L., On the GBDT version of the Bäcklund-Darboux transformation and its applications to linear and nonlinear equations and Weyl theory, Math. Model. Nat. Phenom. 5 (2010), 340-389, arXiv:0909.1537.

[66] Sakhnovich L.A., Problems of factorization and operator identities, Russ. Math. Surv. 41 (1986), no. 1, $1-64$.

[67] Sparano G., Vilasi G., Vinogradov A.M., Vacuum Einstein metrics with bidimensional Killing leaves. I. Local aspects, Differential Geom. Appl. 16 (2002), 95-120, gr-qc/0301020.

[68] Stephani H., Kramer D., MacCallum M., Hoenselaers C., Herlt E., Exact solutions of Einstein's field equations, 2nd ed., Cambridge Monographs on Mathematical Physics, Cambridge University Press, Cambridge, 2003.

[69] Szybka S.J., Stable causality of Black Saturns, J. High Energy Phys. 2011 (2011), no. 5, 052, 9 pages, arXiv:1102.3942.

[70] Tan H.S., Teo E., Multi-black-hole solutions in five dimensions, Phys. Rev. D 68 (2003), 044021, 11 pages, hep-th/0306044.

[71] Tangherlini F.R., Schwarzschild field in $n$ dimensions and the dimensionality of space problem, Nuovo Cimento 27 (1963), 636-651.

[72] Tomimatsu A., Sato H., New exact solution for the gravitational field of a spinning mass, Phys. Rev. Lett. 29 (1972), 1344-1345.

[73] Tomizawa S., Ishihara H., Exact solutions of higher dimensional black holes, arXiv:1104.1468.

[74] Tomizawa S., Morisawa Y., Yasui Y., Vacuum solutions of five dimensional Einstein equations generated by inverse scattering method, Phys. Rev. D 73 (2006), 064009, 8 pages, hep-th/0512252.

[75] Tomizawa S., Nozawa M., Vacuum solutions of five dimensional Einstein equations generated by inverse scattering method. II. Production of the black ring solution, Phys. Rev. D $\mathbf{7 3}$ (2006), 124034, 10 pages, hep-th/0604067.

[76] Tomizawa S., Uchida Y., Shiromizu M., Twist of stationary black hole or ring in five dimensions, Phys. Rev. D 70 (2004), 064020, 5 pages, gr-qc/0405134.

[77] Verdaguer E., Soliton solutions in spacetime with two spacelike Killing fields, Phys. Rep. 229 (1993), 1-80.

[78] Yazadjiev S.S., 5D Einstein-Maxwell solitons and concentric rotating dipole black rings, Phys. Rev. D 78 (2008), 064032, 11 pages, arXiv:0805.1600.

[79] Yazadjiev S.S., Black saturn with a dipole ring, Phys. Rev. D 76 (2007), 064011, 8 pages, arXiv:0705.1840.

[80] Yazadjiev S.S., Completely integrable sector in 5D Einstein-Maxwell gravity and derivation of the dipole black ring solutions, Phys. Rev. D 73 (2006), 104007, 7 pages, hep-th/0602116. 